\title{
Coloured Petri Net-based Traffic Collision Avoidance System encounter model for the analysis of potential induced collisions
}

\author{
Jun Tang ${ }^{\mathrm{a}, \mathrm{b}^{*}}$, Miquel Angel Piera ${ }^{\mathrm{b}}$, Toni Guasch ${ }^{\mathrm{c}}$ \\ ${ }^{a}$ Science and Technology on Information Systems Engineering Laboratory, National University of Defense \\ Technology, Changsha, China \\ ${ }^{\mathrm{b}}$ Department of Telecommunication and System Engineering, Universitat Autònoma de Barcelona, Sabadell, \\ Spain \\ ${ }^{c}$ Department of Systems Engineering and Automation, Universitat Politècnica de Catalunya, Barcelona, Spain \\ jun.tang@e-campus.uab.cat, miquelangel.piera@uab.cat, toni.guasch@upc.edu
}

\begin{abstract}
The Traffic Alert and Collision Avoidance System (TCAS) is a world-wide accepted lastresort means of reducing the probability and frequency of mid-air collisions between aircraft. Unfortunately, it is widely known that in congested airspace, the use of the TCAS may actually lead to induced collisions. Therefore, further research regarding TCAS logic is required. In this paper, an encounter model is formalised to identify all of the potential collision scenarios that can be induced by a resolution advisory that was generated previously by the TCAS without considering the downstream consequences in the surrounding traffic. The existing encounter models focus on checking and validating the potential collisions between trajectories of a specific scenario. In contrast, the innovative approach described in this paper concentrates on quantitative analysis of the different induced collision scenarios that could be reached for a given initial trajectory and a rough specification of the surrounding traffic. This approach provides valuable information at the operational level. Furthermore, the proposed encounter model can be used as a test-bed to evaluate future TCAS logic changes to mitigate potential induced collisions in hot spot volumes. In addition, the encounter model is described by means of the coloured Petri net (CPN) formalism. The resulting state space provides a deep understanding of the cause-and-effect relationship that each TCAS action proposed to avoid an actual collision with a potential new collision in the surrounding traffic. Quantitative simulation results are conducted to validate the proposed encounter model, and the resulting collision scenarios are summarised as valuable information for future air traffic management (ATM) systems.
\end{abstract}

Keywords: TCAS; Encounter model; State space; Potential collision scenario; Petri net

\section{Introduction}

A series of mid-air collisions have occurred over a period of 30 years (1956-1986) (DoT, 2011; NTSB database, 2015). These collisions spurred the Federal Aviation Administration (FAA) to launch the development of an effective collision avoidance system that would act as a last-resort when there is a failure in air traffic controller (ATC)-provided separation services (DoT, 2011). The resulting Traffic Alert and Collision Avoidance System (TCAS) was developed using comprehensive analysis and abundant flight evaluations. The traffic display (which depicts the detailed states of nearby traffic) assists pilots in the visual acquisition of surrounding traffic, providing them time to prepare to manoeuvre the aircraft in the event TCAS advisories are issued. It is particularly important that pilots maintain situational awareness and continue to use good judgment in following TCAS advisories. Maintain frequent outside visual scan, "see and avoid" vigilance, and continue to communicate as needed and as appropriate with ATC (DoT, 2011). This system constitutes a last-resort means, which is accepted worldwide, for effective and significant reduction of the collision probability between aircraft (Netjasov et al., 2013).

TCAS equipped in an aircraft does not control the vehicle directly; the TCAS only issue advisories to pilots on how to manoeuvre vertically to prevent collision. Evidently, the human behaviour (pilot response) has a decisive impact on the collision avoidance (CA) results. However, recorded radar data indicate that pilots do not always behave as assumed by the TCAS logic. The collision of two aircraft in 2002 over Überlingen demonstrated that not anticipating the spectrum of responses limits TCAS's robustness (Johnson, 2004). 
When TCAS is operative on both aircraft that are involved in a one-on-one encounter, each vehicle transmits interrogations to the other via the Mode $\mathrm{S}$ link to ensure collision avoidance (CA) coordination in the process of encounter resolution. TCAS executes independently of ground-based systems, and it relies fully on relevant surveillance equipment on-board the aircraft. TCAS I and its improved version, TCAS II, have been defined and approved by the International Civil Aviation Organisation (ICAO). These versions differ primarily in their alerting capability (DoT, 2011). TCAS I provides traffic advisories (TAs) to assist the pilot in the visual acquisition of intruder aircraft, while TCAS II provides both TAs and resolution advisories (RAs). A TA warns pilots of the potential encounter with neighbouring traffic. A RA is issued to prevent a collision by commanding the pilots to execute an avoidance manoeuvre in the vertical direction. TCAS II version 7.1 is the system that is currently in use. The basic operations are the following (DoT, 2011):

- TCAS broadcasts inquiries and receives answers from neighbouring aircraft to monitor the surrounding airspace constantly.

- TCAS generates a TA when an intruder comes within range of the aircraft and a collision is predicted to occur within 20-48 s (depending on the altitude). TCAS aims to draw the flight crew's attention to the potentially hazardous situation, and it provides a traffic display as well as an audio alert to help the crew to prepare for any resolution manoeuvre that may be required.

- If the situation deteriorates, and a collision is predicted to occur within 15-35 s (depending on the altitude), and TCAS subsequently issues an RA, which is always in the vertical plane. With communication between the TCAS to ensure complementary manoeuvres, the RA could be passive (do not climb, do not descend) or active (climb, descend), depending on the situation. If a RA is issued, then the pilot should respond in a timely and calmly manner (normally the reaction time is set to be $5 \mathrm{~s}$ ) to achieve a safe separation.

- When the threat has passed, TCAS provides a "Clear of Conflict" $(\mathrm{CoC})$ advisory.

The use of the TCAS has had a positive influence on the safety of flights, being effective, beneficial, and significant in reducing the collision probability (Billingsley et al., 2012). According to (DoT, 2011), "TCAS II was designed to operate in traffic densities of up to 0.3 aircraft per square nautical mile (NM), i.e., 24 aircraft within a 5 NM radius, which was the highest traffic density envisioned over the next 20 years". However, there is growing interest in civil applications of Remotely Piloted Aircraft Systems (RPAS) (Wildmann et al., 2014). There is also increasing demand for air travel (Isaacson, 2014). With applications such as surveillance, in which various aircraft would cooperate and compete for a certain target (Yousefi et al., 2013), it is expected that the traffic airspace density will increase considerably in certain small areas during short time periods. These highly congested regions could be regarded as hot spots (Nosedal et al., 2014).

The increased airspace usage can induce a secondary threat as a result of a RA issued by a TCAS, which may issue an inappropriate suggested resolution that resolves a one-on-one encounter with a first threat. Therefore, research that explores such potential collision scenarios is needed to enable Air Traffic Management (ATM) to avoid such accidents.

From 1 January 2005, all civil fixed-wing, turbine-powered aircraft having a maximum take-off mass exceeding $5,700 \mathrm{~kg}$ (ICAO, 2014), or a maximum approved passenger seating configuration of more than 19, are required to be equipped with TCAS II (DoT, 2011), i.e., most but not all aircraft are forced to be equipped with TCAS. Therefore, there would be four equipage/response situations: nonenone (no TCAS on either aircraft), TCAS-none (TCAS on only one aircraft), TCAS-TCAS (TCAS on both aircraft and both follow the advisory), and TCAS-no response (TCAS on both aircraft but only one follows the advisory). Our research purpose is to explore the potential induced collision scenarios resulting from TCAS logic for one-on-one encounters in which the involved aircraft are all TCAS equipped and the pilots climb/descend to resolve threats by perfectly following the TCAS advisories. We utilise the standard model of pilot response to the TCAS advisories. This model involves a 5second pilot response delay followed by a $0.25 \mathrm{~g}$ manoeuvre in compliance with the advisory. Multithreat encounters will not be formalised in the model. The focus of the causal model is on the proper analysis of secondary threats induced by TCAS logic rather than the analysis of TCAS logic in the presence of a secondary threat (Billingsley et al., 2009). 
The subject of this research is the design of an encounter model to identify all of the potential collision scenarios that are representative of possible hazardous situations that may occur in the actual airspace. The dynamics formalised in the model consider the final phases of a collision. Based on aviation safety studies, the final phases generally occur over a period of time of one minute or less. The model is fed the specification of the initial state, which consists only of one aircraft trajectory and the number of aircraft to be considered as surrounding traffic during the experiment. The objective of this paper is to characterise the surrounding traffic scenarios that involve an induced collision.

Data results generated by the model could be processed to provide valuable information at the operational level for future ATM scenarios. The results are summarised as a set of potential collision scenarios that could be used not only to help ATCs to recognise those traffic scenarios in which a potential induced collision exists but also to assist the pilots with predictive information regarding potential future surrounding traffic during the flight leading to encounters. Therefore, this approach would be highly valuable for the operations of ATCs and pilots in the future hectic and congested traffic to improve the flight safety.

- Aviation safety studies: The state space allows for the traceability of the sequence of TCAS proposed actions that address an induced collision with the surrounding traffic. Furthermore, causal analysis of these induced collisions could provide a baseline the design of new TCAS logic rules to mitigate any undesirable effects (Billingsley et al., 2009).

- ATC: During the flight execution phase, the generated TCAS state space of all possible induced collision scenarios can constitute a supporting database that interlink pattern recognition tools (Carvalho et al., 2009) to recognise those traffic scenarios that may degenerate into a collision. An advanced warning could be automatically displayed in the ATC visualisation tools when the traffic in a particular airspace volume matches one of the scenarios generated by this model, depicted in Fig. 1 .

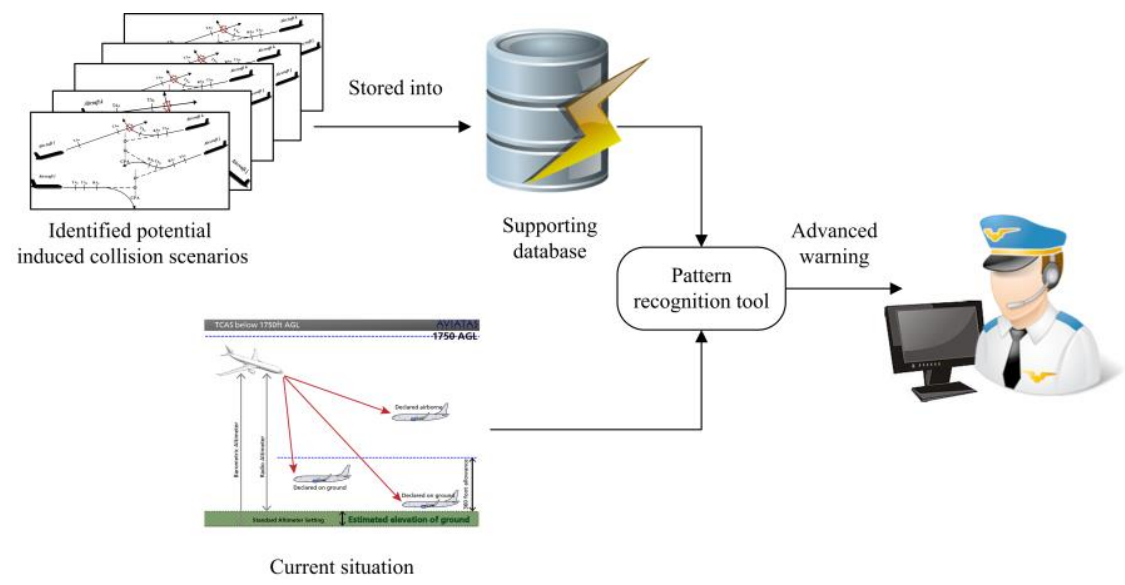

Figure 1. Pattern recognition for potential induced collision scenarios

- Pilots: Achieving adequate separation to resolve encounters is also contingent on the pilot's workload in the short CA period. The identified potential collision scenarios should be used in the flight route re-planner to help the ATC manage air traffic trajectories to ensure a safe flight, thereby reducing the encounter probability while partly reducing the pilots' workload during the execution phase. In addition, this approach helps the pilots perceive all of the possible future situations, especially the potential collision scenarios in the high-density traffic, and this could decrease threat frequency.

- ATM: There are several studies (Catena et al., 2014; Conde et al., 2012; Brooker, 2013) that analyse the introduction of RPAS in a non-segregated airspace. The proposed model could contribute to amend some RPAS trajectories without increasing the airspace latent capacity.

This research discusses pairwise-aircraft threats in which multiple induced threats are resolved sequentially in pairs. In a pairwise approach, if one threat solution induces a new threat, then the original solution may be modified until a threat-free solution is found. This approach is effective but could also potentially fail in certain situations (Kuchar and Yang, 2000). The proposed causal model has been developed with the assumption of four-dimensional (4D) trajectories (different three- 
dimensional (3D) positions of an aircraft in different discrete time steps), TCAS II equipped aircraft and en-route traffic. The induced potential collision discussed in this paper is that TCAS may issue an improper manoeuvre, which resolves the encounter with the first threat, but induces a collision with a second threat or is incompatible with the second threat. Note that this is different from the term "multithreat", which is used to identify an encounter between a TCAS-equipped aircraft and simultaneously with more than one intruder aircraft (Billingsley et al., 2009).

In addition, uncertainty in the compliance of the pilot to advisories makes the collision avoidance logic challenging, with even a single threat between two aircraft possibly causing a collision. The current causal model does not explicitly consider variability in the pilot response. Instead, the model only uses a deterministic model to predict the future aircraft trajectories. If the model is improved with probabilistic pilot response models, then different probabilistic collisions could be generated and analysed, in an effort to compute the severity of each uncertainty considering particular surrounding traffic.

A brief outline of the remainder of this paper is as follows. Section 2 summarises the existing encounter models and presents the motivation for state space analysis. Section 3 provides a detailed description of the TCAS logic. Section 4 introduces the CPN formalism. Section 5 explains the generation process of the potential collisions and depicts the proposed causal model. Section 6 illustrates the results and analysis of the typical collision scenarios. Finally, the conclusions and future work are presented in Section 7.

\section{Motivation}

For two aircraft that are both equipped with TCAS (TCAS-TCAS situation), when a one-on-one encounter is declared, a two-step process is used to select the cooperative RA for the threat geometry. The first step is to decide the appropriate sense (upward or downward) for each aircraft involved. Based on the range and altitude tracks of the involved aircraft, the TCAS logic models their flight paths from their current positions to the closest point of approach (CPA). Next, TCAS selects an opposite sense RA for each aircraft to determine which sense provides the most vertical separation at CPA (DoT, 2011), shown in Fig. 2. In the encounter, the downward sense for Aircraft $i$ along with the upward sense for Aircraft $j$ will be selected because these non-crossing senses provide greater vertical separation. The second step is to determine the RA strength, which is the least disruptive to the existing flight paths while still providing at least the Altitude Limit (ALIM) of vertical separation between the two involved aircraft at the CPA. In other words, the amendment of the vertical speed should be minimal.

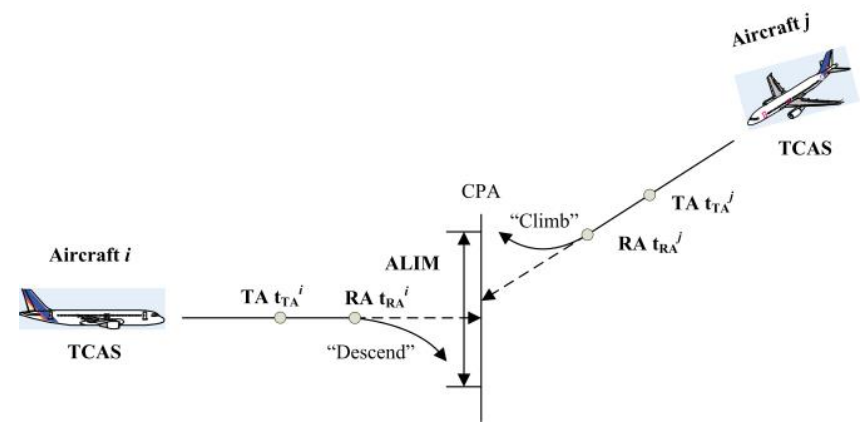

Figure 2. TCAS coordination

The range and altitude tests are implemented on each neighbouring intruder. If the time to the CPA in both the horizontal and vertical planes meet the temporal threshold and/or the spatial threshold for protected airspace (distance modification (DMOD) and altitude threshold (ZTHR)) in slowclosure-rate encounters (time criteria values are not appropriate), the intruder is declared to be a threat (DoT, 2011). These temporal and spatial values vary with different sensitivity levels (SLs). The values used to issue TAs and RAs are shown in Table 1 (DoT, 2011). In addition, the ALIM provides the desired vertical minimum separation at the CPA. 
Table 1. Sensitivity level and threshold values

\begin{tabular}{|c|c|c|c|c|c|c|c|c|}
\hline \multirow{2}{*}{$\begin{array}{c}\text { Own Altitude } \\
\text { (feet) }\end{array}$} & \multirow{2}{*}{ SL } & \multicolumn{2}{|c|}{$\begin{array}{c}\text { Time } \\
\text { (second) }\end{array}$} & \multicolumn{2}{c|}{$\begin{array}{c}\text { DMOD } \\
\text { (NM) }\end{array}$} & \multicolumn{2}{c|}{$\begin{array}{c}\text { ZTHR } \\
\text { (feet) }\end{array}$} & $\begin{array}{c}\text { ALIM } \\
\text { (feet) }\end{array}$ \\
\cline { 3 - 9 } & & TA & RA & TA & RA & TA & RA & RA \\
\hline$<1000$ & 2 & 20 & N/A & 0.30 & N/A & 850 & N/A & N/A \\
\hline $1000-2350$ & 3 & 25 & 15 & 0.33 & 0.20 & 850 & 600 & 300 \\
\hline $2350-5000$ & 4 & 30 & 20 & 0.48 & 0.35 & 850 & 600 & 300 \\
\hline $5000-10000$ & 5 & 40 & 25 & 0.75 & 0.55 & 850 & 600 & 350 \\
\hline $10000-20000$ & 6 & 45 & 30 & 1.00 & 0.80 & 850 & 600 & 400 \\
\hline $20000-42000$ & 7 & 48 & 35 & 1.30 & 1.10 & 850 & 700 & 600 \\
\hline$>42000$ & 7 & 48 & 35 & 1.30 & 1.10 & 1200 & 800 & 700 \\
\hline
\end{tabular}

The scenario shown in Fig. 3 illustrates the process of an induced collision occurrence in which TCAS would fail. The scenario consists of three aircraft that are all in the sixth SL: Aircraft 1 is cruising at flight level (FL) 145; Aircraft 2 is descending from FL160; and Aircraft 3 is climbing slightly from FL148. All aircraft are fully equipped with TCASs. These systems incessantly survey the surrounding airspace by sending inquiries and receiving responses from neighbouring aircraft. Thus, when Aircraft 2 flies into the range (time) of Aircraft 1, a TA is issued by TCAS to warn the crew of Aircraft 1 that an encounter is predicted to occur within $t_{T A}^{1}$. Next, a RA at $t_{T A}^{1}$ is issued to provide a suggestion to the pilot. To resolve the detected threat, Aircraft 2 performs a climb operation, while Aircraft 1 descends to provide the greatest vertical separation at CPA. Normally, the RA strength selects the ALIM as the lowest safe separation that leads to the minimum change of vertical speed. Meanwhile, a new threat is detected between Aircraft 1 and Aircraft 3 because of their closing trend in the horizontal plane.

The TAs in both of the aircraft (Aircraft 1 and Aircraft 3) send announcements to the pilots. However, the RAs would not be triggered because the aircraft are travelling in opposite directions in the vertical plane, and the situation does not deteriorate. Originally, there was no threat between Aircraft 3 and Aircraft 2. However, the climbing of Aircraft 2 induces an encounter event with the slight climbing Aircraft 3, which is locked into its trajectory by Aircraft 1 (there would be a predictive collision if Aircraft 3 descends based on their current flight states). Unfortunately, due to the time concurrency of the different actions and the loss of minimal collision separation, a collision could occur as a consequence of previous decisions and the extraordinary geometry.

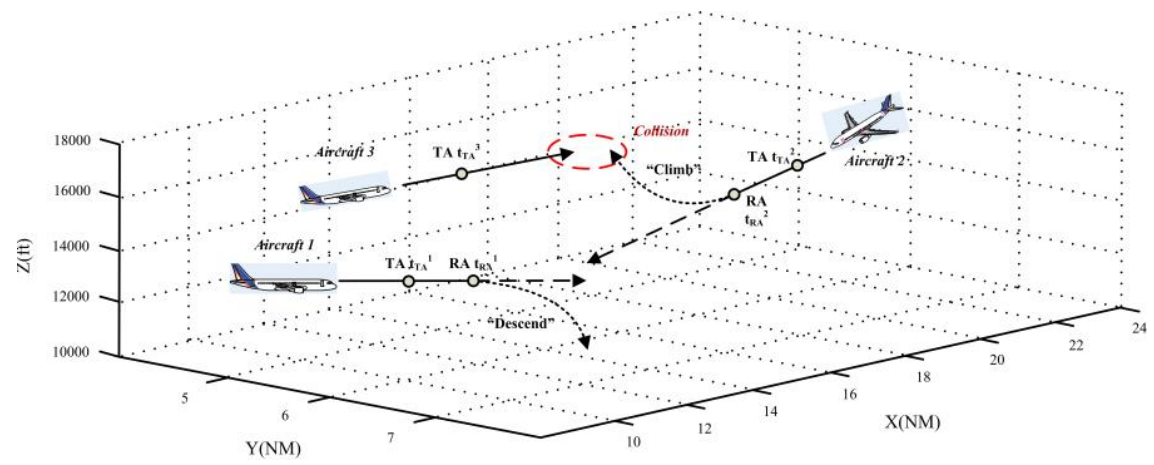

Figure 3. Three-aircraft scenario

These potential collision situations are notably rare for current traffic densities (less than 0.3 aircraft per square NM). However, the likelihood of such situations will increase as the airspace usage increases. Thus, for a hot spot with the surrounding traffic, different cooperative sense selections for resolving a one-on-one encounter would initiate different states that must be analysed to discern the cause-and-effect relationships and to explore the potential induced collision states.

Current Air Traffic Management research programs (i.e., Single European Sky ATM Research (SESAR) and next generation air transportation systems (NextGen)) attempt to overcome airspace capacity shortages while improving cost-efficient operations and safety (Tang et al., 2014). Air Traffic 
Flow and Capacity Management (ATFCM) was established to utilise the European airspace capacity to the maximum extent possible while enabling safe, orderly and expeditious traffic flows. However, current ATFCM actions do not ensure traffic separation/synchronisation at an individual level (Ruiz, 2013). The development and application of the Global Navigation Satellite System (GNSS) and the Automatic Dependent Surveillance Broadcast (ADS-B) system (McCallie et al., 2011) enable aircraft to obtain highly accurate positional and directional information regarding themselves and other nearby aircraft. Thus, several efforts (Nosedal et al., 2014; Ruiz, 2013) have been made to develop trajectorybased operations (TBOs) that focus on flight efficiency, predictability, environment and capacity to construct a trajectory-based ATM system. In such a system, the partners optimise their trajectories through common 4D trajectory information, also known as reference business trajectories (RBTs). The induced collision scenarios can be generated off-line (by the proposed model) and then stored in a database. These identified scenarios could then be used for the development of innovative tools to address future congested traffic scenarios while improving the safety of flight.

- $\quad$ Strategic: The highly efficient computational performance achieved by the algorithms (Nosedal et al., 2014; Ruiz, 2013) could be useful for the integration of Decision Support Tools (DSTs) designed for the ATFCM. These tools could help to identify geographical areas that may have high probabilities of becoming unstable (i.e., hot spots) and to locate "highly congested regions" (e.g., a high number of conflicts predicted to occur in a same spatiotemporal region). Considering the various sources of effecting factors (especially weather conditions) that can affect the RBTs, new tools could be deployed to compute the probability of trajectories programmed in the hot-spots that could degenerate and match any scenario in the induced collision database, thereby providing an auxiliary support in the analysis of hot spots.

- Tactical: During the flight execution phase, the database of all potential induced collision scenarios can be directly related to pattern recognition. Proper automation contrast can be used to evaluate situations in which multiple aircraft are involved. Thus, the database can help us to evaluate the deviation of the proposed trajectories, which could lead to hazardous scenarios (existing potential induced collisions) within a foreseen time-window of 10 to 20 min. This time window could provide sufficient time to allow the ATC to resolve any hazardous deviation.

- Operational: Surveillance technologies (e.g., ADS-B) are fully operative in all aircraft, enabling the Airborne Separation Assurance System (ASAS) (Netjasov et al., 2013) to provide the precise self-separation and synchronisation of aircraft. The pattern (a potential induced collision scenario) that fits the current situation can provide relevant information to the pilots. This approach enhances the ASAS at the operational level in high-density traffic scenarios (without the need to heighten or change the relevant logic) to enable precise monitoring of all of the traffic to assure safe and efficient operations.

The potential collision scenarios identified by the proposed causal model are compatible with the current surveillance and management of threats, as well as with the on-board TCAS. Thus, the safety of air traffic can be ensured by three different layers of threat management (i.e., strategic, tactical and operational). This approach may reduce the negative impact of effecting factors on the safety levels of the current ATM.

\subsection{Encounter model overview}

Several encounter models based on different methods and techniques have been developed over the years to support the certification and performance analysis of TCAS. These models are used to generate encounter situations for use in estimating the rate of mid-air collisions events; in these models, aircraft are treated as point masses.

It is necessary to examine the TCAS safety issues that motivated the development of encounter models. In Kochenderfer et al. (2010), they described a methodology for producing an encounter 
model construction based on a Bayesian statistical framework, and they used the model to evaluate the safety of collision avoidance systems for manned and unmanned aircraft. Kuchar et al. (2004) attempted to use a fault tree to model the outer-loop system failures or events that, in turn, define the environment for a fast-time Monte Carlo inner-loop simulation of a close encounter. Zeitlin et al. (2006) outlined the steps of a safety analysis process to assess the performance of TCAS on conventional and unconventional aircraft. Espindle et al. (2009) constructed the U.S. correlated encounter model utilising importance sampling techniques to increase the precision of the results and to evaluate the safety impact of the latest TCAS (version 7.1). Netjasov et al. (2010) proposed an encounter model that contains the technical, human and procedural elements of TCAS operations. The model was demonstrated to work well for a historical en-route mid-air collision event (Department of Trade, 1982), and the model was powerful in determining the most critical elements that contribute to non-zero collision probability in TCAS operations. Several other researchers focused on the pilot behaviour that could influence the safety risk. Lee and Wolpert (2012) combined Bayes nets and game theory to predict the behaviour of hybrid systems involving both humans and automated components, thereby predicting aircraft pilot behaviour in potential mid-air collision situations. Chryssanthacopoulos and Kochenderfer (2011) extended the pilot response model in which the pilot responded deterministically to all alerts to include probabilistic pilot response models that capture the variability of pilot reaction time to enhance robustness. Garcia-Chico and Corker (2007) provided a detailed analysis of the human operational errors that would increase the probability of a collision.

Of special relevance is the Interactive Collision Avoidance Simulator (InCAS, developed by EuroControl) (ICAO, 2006). InCAS is a software tool that is TCAS logic-based, and it is designed for the replay of a real or a synthetic event. InCAS is an interactive system for the evaluation, study, demonstration and training on TCAS, and it is designed to simulate incidents that provide a relatively exact reconstruction of reality. Although it is not a standard encounter model for the support of the safety assessment of TCAS operations (ICAO, 2006), InCAS provides valuable information and data for operational understanding and also for pilot TCAS training.

The input data of the existing models are the known information of several trajectories. Therefore, the models could be used to determine whether a multi-aircraft scenario contains a potential collision or not. However, there is a lack of rigorous models to identify and generate all of the potential induced collision scenarios for a certain number of aircraft in a particular hot spot area.

\subsection{State space analysis}

There are several formalisms to explore the dynamics of discrete systems, such as an automaton, Markov chain, Timed automata, Petri Nets (PN), coloured Petri net (CPN), min-max algebra, etc. (the formalisms are summarised in Kristensen and Jensen (2004). Of these formalisms, the PN and CPN formalisms are versatile and well-founded modelling languages that can be used in practice for systems of the size and complexity found in real industrial applications (Jensen, 2009). CPN is a graphical and discrete-event language that combines the capabilities of PN with the capabilities of a high-level programming language. Petri nets provide the foundation of the graphical notation and the basic primitives for modelling concurrency, communication, and synchronisation toward a very broad class of systems. However, these nets are intended to be a general modelling language, i.e., it is not aiming to model a specific class of systems. Both PN and CPN have been employed to describe the synchronisation of concurrent processes. However, CPN provides the strength that is required to define data types and manipulate data values (Salimifard and Wright, 2001). CPN has been used to verify and validate systems through property analysis. More recently, the state space analysis tool has been used to explore the dynamic evolution of a system and to determine all of the possible future states that are reachable from a given current state vector (initial trajectories in this research).

TCAS is a hybrid system in the sense of informatics, and its components and logic can be analysed using discrete system techniques (Ladkin, 2004). These techniques can model the operation of TCAS as a discrete sequence of events in time; each event occurs at a particular instant in time and causes a change of system state. In addition, although the widespread system has been in application with new developments for more than 30 years, essential parts of its causal analysis, especially those for potential induced collision scenarios that could be considered to be TCAS failures, apparently have not yet been performed, which fits the definition of a state space that contains all of the possible 
occurrence sequences and states that can be reached from an initial (known) state. In Tang et al. (2014), a CPN model is introduced as a key approach to analyse the state space of a congested traffic scenario in which the events that could drive an encounter into a collision are explored. This model provides a useful tool for better understanding the surrounding traffic conditions (both at macro- and micro-levels) that could lead to a collision and also to check for future TCAS logic updates. In this research, the TCAS logic was modelled to analyse the cause-and-effect relationships between successive events (separation minima lost, RAs, TAs, manoeuvres) that produce a phenomenon (state of the system). This approach corresponds to the central concept of PN and the enhanced version, CPN (Piera and Music, 2011).

For a flight scenario, the causal model has been demonstrated to be highly useful for checking a variety of state space situations. This determination is accomplished by means of a reachability tree (also called occurrence graph), in which the different states that can be reached from an initial state can be generated together with the events that caused the state change. This approach enables understanding of the cause-effect relationship of each action and how the effects of an action are propagated upstream and downstream through the different actions (Tang et al., 2014). In essence, the analysis problem becomes a search problem in which those organisations responsible for air traffic safety can determine the initial aircraft trajectories and the Airborne Collision Avoidance System (ACAS) induced manoeuvres that may lead to a failure of resolving the conflicts.

\section{Mathematical description of TCAS operations}

This section specifies the mathematical model for the TCAS II algorithm that is potentially used in a one-on-one encounter with both aircraft being TCAS equipped. In general, during normal flight, the aircraft receives instructions from ATC and is flying in accordance with the instructions. Meanwhile, TCAS is constantly surveying the surrounding airspace, broadcasting interrogations and receiving responses from nearby aircraft. Though aircraft mostly fly in the pre-set trajectories, various sources of uncertainty, such as the influence of the weather conditions (especially the wind), the aircraft control systems (both the pilot and aircraft performance errors) and the positioning/tracking precision (even considering the more precise navigation systems), affect the aircraft while flying their trajectories in a precise and straight way. The separation between aircraft may decrease and the TCAS logic would take effect when the TA/RA criteria are met. The mathematical description of TCAS logic is primarily based on (DoT, 2011).

An aircraft can be modeled as a unique point in the space surrounded by a 3D safety volume shape, thus a threat is considered to be resolved by the TCAS when the time and/or the spatial threshold of both aircraft overlap, while TCAS logic fails to avoid the collision when the $\mathrm{H}_{\mathrm{cl}}$ and $\mathrm{D}_{\mathrm{cl}}$ volumes of both aircraft overlap. Normally, $D_{\mathrm{cl}}$ is double the average length of the aircraft fleet operating in the basic volume (or wingspan if longer) while $\mathrm{H}_{\mathrm{cl}}$ is double the average height of the aircraft fleet operating in the basic volume. The values of $\mathrm{H}_{\mathrm{cl}}$ and $\mathrm{D}_{\mathrm{cl}}$ can be obtained from the EuroControl reference (EuroControl, 2001).

\subsection{Threat detection algorithm}

Given that TCAS executes with local scope and within a short period, the hot spot to be analysed by the proposed model will be called basic volume. This can be regarded as a Euclidean 3D space (not a curved space) (Ruiz, 2013) whose definition criteria could be based on the range of reliable surveillance that the aircraft supports (generally 14 NM (DoT, 2011)). Euclidean spaces make the construction of the basic volume simpler. Thus, a planar projection of the Earth has been considered by using a Cartesian coordinate system (Netjasov et al., 2013) and with a minimum distortion (Ruiz, 2013). The region formed by $x$ and $y$ axes indicates the horizontal plane, and $z$ stands for the flight level. For Aircrafti $(i=1,2, \ldots, n)$, its dynamic characteristics can be described as (Netjasov et al., 2010): 


$$
\begin{aligned}
& p_{t}^{i}=\left[\begin{array}{c}
x_{t}^{i} \\
y_{t}^{i} \\
z_{t}^{i}
\end{array}\right], \quad v_{t}^{i}=\frac{d p_{t}^{i}}{d t}=\left[\begin{array}{c}
v_{t}^{i} \cos \gamma_{t}^{i} \cos \theta_{t}^{i} \\
v_{t}^{i} \cos \gamma_{t}^{i} \sin \theta_{t}^{i} \\
v_{t}^{i} \sin \gamma_{t}^{i}
\end{array}\right]=\left[\begin{array}{c}
v_{t, x}^{i} \\
v_{t, y}^{i} \\
v_{t, z}^{i}
\end{array}\right] \\
& 0<\theta_{t}^{i}<2 \pi, \quad-\frac{\pi}{2} \leq \gamma_{t}^{i} \leq \frac{\pi}{2}
\end{aligned}
$$

The formula defines $p_{t}^{i}$ and $v_{t}^{i}$ as the position and speed, respectively, of Aircrafti in 3D. Let $\theta_{t}^{i}$ represents the course angle. This is the orientation of speed $v_{t}^{i}$ in the $\mathrm{x}-\mathrm{y}$ plane (measured from the $\mathrm{x}$ axis in a counter-clockwise direction). Let $\gamma_{t}^{i}$ designate the angle of climb. This is the orientation of speed $v_{t}^{i}$ in the vertical plane (measured from the horizontal plane with up as positive and down as negative). Let $p_{t, h}^{i}=\left(x_{t}^{i}, y_{t}^{i}\right)^{T}$ and $v_{t, h}^{i}=\left(v_{t, x}^{i}, v_{t, y}^{i}\right)^{T}$ be the position and the speed, respectively, of Aircraft $i$ in the horizontal plane and analogously for Aircraft $j$.

Depending on the geometry of the encounter, an RA may be delayed or not selected at all if the CPA does not meet the DMOD and ZTHR threshold of RA in the corresponding SL. Thus, an efficacious threat that would deteriorate to initiate the RA can be detected based on the following set of equations.

$$
\begin{aligned}
& T_{t_{C P A}, h}^{i j}=\frac{-\sqrt{\left(x_{t}^{i}-x_{t}^{j}\right)^{2}+\left(y_{t}^{i}-y_{t}^{j}\right)^{2}}}{\sqrt{\left(v_{t, x}^{i}-v_{t, x}^{j}\right)^{2}+\left(v_{t, y}^{i}-v_{t, y}^{j}\right)^{2}} \cdot \cos \left(\alpha_{t}^{i j}-\beta_{t}^{i j}\right)} \\
& \cos \alpha_{t}^{i j}=\arctan \left(\frac{v_{t, x}^{i}-v_{t, x}^{j}}{\left.v_{t, y}^{i}-v_{t, y}^{j}\right)}\right. \\
& \cos \beta_{t}^{i j}=\arctan \left(\frac{x_{t}^{i}-x_{t}^{j}}{y_{t}^{i}-y_{t}^{j}}\right) \\
& T_{t_{C P A}, z}^{i j}=-\frac{z_{t}^{i}-z_{t}^{j}}{v_{t, z}^{i}-v_{t, z}^{j}} \\
& D_{t, h}^{i j}=\sqrt{\left(x_{t}^{i}-x_{t}^{j}\right)^{2}+\left(y_{t}^{i}-y_{t}^{j}\right)^{2}} \\
& D_{t, z}^{i j}=\sqrt{z_{t}^{i}-z_{t}^{j} \mid} \\
& D_{t_{C P A}, h}^{i j}=\sqrt{\left[\left(x_{t}^{i}+v_{t, x}^{i} \cdot T_{t_{C P A}, z}^{i j}\right)^{2}-\left(x_{t}^{j}+v_{t, x}^{j} \cdot T_{t_{C P A}, z}^{i j}\right)^{2}\right]+\left[\left(y_{t}^{i}+v_{t, y}^{i} \cdot T_{t_{C P A}, z}^{i j}\right)^{2}-\left(y_{t}^{j}+v_{t, y}^{j} \cdot T_{t_{C P A}, z}^{i j}\right)^{2}\right]} \\
& D_{t_{C P A}, z}^{i j}=\sqrt{\left[\left(z_{t}^{i}+v_{t, z}^{i} \cdot T_{t_{C A A}, h}^{i j}\right)^{2}-\left(z_{t}^{j}+v_{t, z}^{j} \cdot T_{t_{C P A}, h}^{i j}\right)^{2}\right]}
\end{aligned}
$$

In these above equations, $T_{t_{C P A}, h}^{i j}$ and $T_{t_{C P A}, z}^{i j}$ are defined as the time to CPA in the horizontal and vertical planes between Aircrafti and Aircraft $j$ at time t. The calculation formulas are discussed in (Netjasov et al., 2013). The symbols $\alpha_{t}^{i j}$ and $\beta_{t}^{i j}$ refer to the direction angle of the speed and position difference vectors, respectively, and $\alpha_{t}^{i j}-\beta_{t}^{i j} \neq \pm \pi / 2 . D_{t, h}^{i j}$ and $D_{t, z}^{i j}$ are the horizontal and vertical distances between Aircrafti and Aircraft $j$ at time t. $D_{t_{C P A}, h}^{i j}$ is the horizontal distance between Aircraft $i$ and Aircraft $j$ at CPA in the horizontal plane, and $D_{t_{C A A}, z}^{i j}$ is the vertical distance between Aircrafti and Aircraft $j$ at CPA in the vertical plane. In factual situations, not all approaching aircraft would initiate the resolution measures because sometimes their CPAs are not in the scope of the RA criteria. Thus, a TA that will deteriorate to RA should be fired when the following set of conditions is met:

$$
\left(0<T_{t_{C A}, h}^{i j}<\operatorname{Time}_{T A}\right) \wedge\left(0<T_{t_{C P A}, z}^{i j}<\operatorname{Time}_{T A}\right) \wedge\left(D_{t_{C P A}, h}^{i j}<D M O D_{R A}\right) \wedge\left(D_{t_{C P A}, z}^{i j}<Z T H R_{R A}\right)
$$


In the case of a slow closure encounter due to the spatial values of TA and RA being slightly different, the threat can be detected when the horizontal and vertical distances between Aircraft $i$ and Aircraft $j$ at time $t$ satisfy the spatial criteria:

$$
\left(D_{t, h}^{i j}<D M O D_{T A}\right) \wedge\left(D_{t, z}^{i j}<Z T H R_{T A}\right)
$$

Note that both the time and spatial thresholds of TA and RA depend on the SL (altitude), and these are provided in Table 1.

\subsection{Threat resolution algorithm}

If the following alternative set of conditions is satisfied, an RA will be issued.

$$
\left(0<T_{t, h}^{i j}<\text { Time }_{R A}\right) \wedge\left(0<T_{t, z}^{i j}<\text { Time }_{R A}\right)
$$

or

$$
\left(D_{t, h}^{i j}<D M O D_{R A}\right) \wedge\left(D_{t, z}^{i j}<Z T H R_{R A}\right)
$$

where Time $_{R A}$ is the time to the CPA threshold value for RA. $D M O D_{R A}$ and $Z T H R_{R A}$ are the range and altitude limit values, respectively, for RA issuance in the case of slow-closure-rate encounters when the time threshold values are not feasible. A two-step process is used to select the appropriate RA for the threat geometry when the RA is issued. First, the RA sense (upward/downward) is set based on the predictive altitude at CPA of the horizontal plane. This can be calculated using the following expressions.

$$
\begin{aligned}
& z_{t_{C P A}}^{i}=z_{t}^{i}+v_{t, z}^{i} \cdot T_{t_{C P A}, h}^{i j} \\
& z_{t_{C P A}}^{j}=z_{t}^{j}+v_{t, z}^{j} \cdot T_{t_{C P A}, h}^{i j}
\end{aligned}
$$

First, TCAS is to determine the resolution sense that is based on the value of $z_{t_{C P A}}^{i}$ and $z_{t_{C P A}}^{j}$. Then, the higher one turns upward while the other turns downward. Then, TCAS is designed to select the strength of the advisory that is the least disruptive to the existing flight path while still providing ALIM vertical separation between Aircrafti and Aircraft $j$ at CPA. Note that in modelling aircraft response to RAs, the expectation is that the pilot will begin the initial $a_{0}=0.25 \mathrm{~g}$ acceleration manoeuvre within 5 seconds (DoT, 2011). Therefore, in this research, when an encounter occurs, both of the aircraft accelerate to appropriate speeds with opposite acceleration $(0.25 \mathrm{~g}$ and $-0.25 \mathrm{~g})$ in the vertical plane, and the response time of the pilot is assumed to be 5 seconds.

For Aircraft $i$, the appropriate amendment strength should be:

$$
\Delta_{t, z}^{i}=A L I M_{R A}+z_{t C P A}^{i}-z_{t C A}^{j}
$$

Additionally, the acceleration time $t_{a_{0}}^{i}$ of Aircraft $i$ can be calculated by this equality:

$$
\Delta_{t, z}^{i}=\frac{a_{0} t_{a_{0}}^{i 2}}{2}+a_{0} t_{a_{0}}^{i}\left(T_{t_{C P A}, h}^{i j}-t_{a_{0}}^{i}\right)
$$

Thus,

$$
t_{a_{0}}^{i}=T_{t_{C P A}, h}^{i j}-\sqrt{T_{t_{C P A}, h}^{i j}-2 \Delta_{t, z}^{i} / a_{0}}
$$

Based on fairness, the acceleration time $t_{a_{0}}^{j}$ of Aircraft $j$ should be equal to $t_{a_{0}}^{i}$.

\section{CPN formalism}

CPN formalism supports several quantitative and qualitative methods for the analysis of the system dynamics. There are several examples of CPN models (Salimifard and Wright, 2001; Ladkin, 2004; Zuniga et al., 2013; Piera et al., 2014) developed by the simulation community using quantitative approaches. The CPN method has characteristics that allow modelling of true concurrency, parallelism 
or conflicting situations that are present in dynamic systems. The formalism allows for the specification of dynamic discrete-event oriented models without ambiguity and also for modelling the information flow. This is an important characteristic that can be very useful for decision making.

The semantics of the modelling formalism CPN can be defined as the tuple (Jensen, 1993):

$$
C P N=(\Sigma, P, T, A, N, C, G, E, I)
$$

where

- $\Sigma=\left\{\mathrm{C} 1, \mathrm{C} 2, \ldots, \mathrm{C}_{\mathrm{nc}}\right\}$ represent the finite and not-empty set of colours. They allow the attribute specification of each modelled entity.

- $\mathrm{P}=\left\{\mathrm{P} 1, \mathrm{P} 2, \ldots, \mathrm{P}_{\mathrm{np}}\right\}$ represent the finite set of place nodes.

- $\mathrm{T}=\left\{\mathrm{T} 1, \mathrm{~T} 2, \ldots, \mathrm{T}_{\mathrm{nt}}\right\}$ represent the set of transition nodes such that $\mathrm{P} \cap \mathrm{T}=\varnothing$, which are normally associated activities in the real system.

- $\mathrm{A}=\left\{\mathrm{A} 1, \mathrm{~A} 2, \ldots, \mathrm{A}_{\text {na }}\right\}$ represent the directed arc set, which relate transition and place nodes such as $\mathrm{A} \subseteq \mathrm{P} \times \mathrm{T} \cup \mathrm{T} \times \mathrm{P}$

- $\mathrm{N}=$ the node function $\mathrm{N}\left(\mathrm{A}_{\mathrm{i}}\right)$, which is associated with the input and output arcs. If one is a place node, then the other must be a transition node and vice versa.

- $\mathrm{C}=$ the colour set functions, $\mathrm{C}\left(\mathrm{P}_{\mathrm{i}}\right)$, which specify the combination of colours for each place node, such as $\mathrm{C}: \mathrm{P} \rightarrow \sum$.

- $\mathrm{G}=$ Guard function, which is associated with transition nodes, $\mathrm{G}\left(\mathrm{T}_{\mathrm{i}}\right), \mathrm{G}: \mathrm{T} \rightarrow$ EXPR. This is normally used to inhibit the event associated with the transition upon the attribute values of the processed entities. If the processed entities satisfy the arc expression but not the guard, the transition will not be enabled.

- $\mathrm{E}=$ the arc expressions $\mathrm{E}\left(\mathrm{A}_{\mathrm{i}}\right)$, such as $\mathrm{E}: \mathrm{A} \rightarrow \mathrm{EXPR}$. For the input arcs, they specify the quantity and type of entities that can be selected among the ones present in the place node to enable the transition. When dealing with an output place, these expressions specify the values of the output tokens for the state generated when the transition fires.

- $I$ = Initialization function $I\left(\mathrm{P}_{\mathrm{i}}\right)$, which allows the value specification for the initial entities in the place nodes at the beginning of the simulation. This is the initial state for a particular scenario.

- EXPR denotes logic expressions provided by any inscription language (logic, functional, etc.).

- The state of every CPN model is also called the marking and is composed of the expressions associated with each place p. These must be closed expressions, i.e., they cannot have any free variables.

A model can be graphically represented by circles (called place nodes), rectangles or solid lines (called transition nodes) and directed arrows (called arcs) that connect a transition with a place node or a place node with a transition. To model the occurrences of activities, the input place nodes to a transition node must hold at least the same number of entities (called tokens) as the correspondent arc weight. The colours of the potential tokens must satisfy the expressions associated with the colours in the arc expressions. The Boolean condition attached to the transition (guard) must be the final restriction that must be fulfilled for the transition to occur. When all of the latter conditions are satisfied, the transition can be "fired". This means that the entities that satisfy the mentioned conditions are destroyed from the original input place nodes and new entities (i.e., tokens) are created in the output place nodes of the transition. The new tokens are created with the characteristics and quantities stated in the colours and output arc weights, respectively.

Traditionally, the place nodes are used to model resource availability or logic conditions that need to be satisfied. The transition nodes can be associated with activities or events to be executed.

One of the most powerful quantitative analysis tools of CPN is the reachability tree. The goal of the reachability tree is to find all of the markings that can be reached from a certain initial system state, representing a new system state at each tree node and a transition firing in each arc. The reachability tree allows the following:

- All of the manoeuvres that can be issued by TCAS consider the air traffic scenario (markings) which can be reached starting from a certain set of initial system conditions (traffic scenario).

- The transition sequence is fired to drive the system from a certain initial state to a particular state of interest. In this paper, end-states are those states in which a collision occurs due to either the 
inability of ACAS to resolve the situation, or alternatively, an internal failure within the individual ACAS to provide what would otherwise have been a reasonable RA.

\section{Causal encounter model in CPN formalism}

This section considers a proposed novel generation process of potential collision scenarios and constructs the encounter model using CPN formalism.

\subsection{Scenario generation process}

This section describes the processing required to drive an aircraft with a known trajectory into various induced collision scenarios consisting of multiple aircraft equipped with TCAS. The initial conditions (i.e., input data and parameters) of the proposed model are the aircraft trajectory and the amount of intruder aircraft. Based on the threat geometry with the known aircraft, the state of neighbouring aircraft is generated through the following step-by-step process: calculate the CPA for a threat aircraft, and then characterise its speed and position feasible values. The detailed explanation of the process is depicted in Fig. 4 and Fig. 5. Each threat consists of a pair of aircraft trajectories. Although induced threats are rare in the actual airspace, simulating them is a straightforward extension (Billingsley et al., 2009). The causal encounter model that is proposed in this paper generates all of the potential induced collision scenarios for multiple aircraft based on the initial state of a representative aircraft. These scenarios include the initial airspeed and position at the corresponding time, together with a specification of the number of aircraft to be considered as the surrounding traffic in the hot spot. The process used for generation of the induced collision scenarios is outlined in Fig. 4.

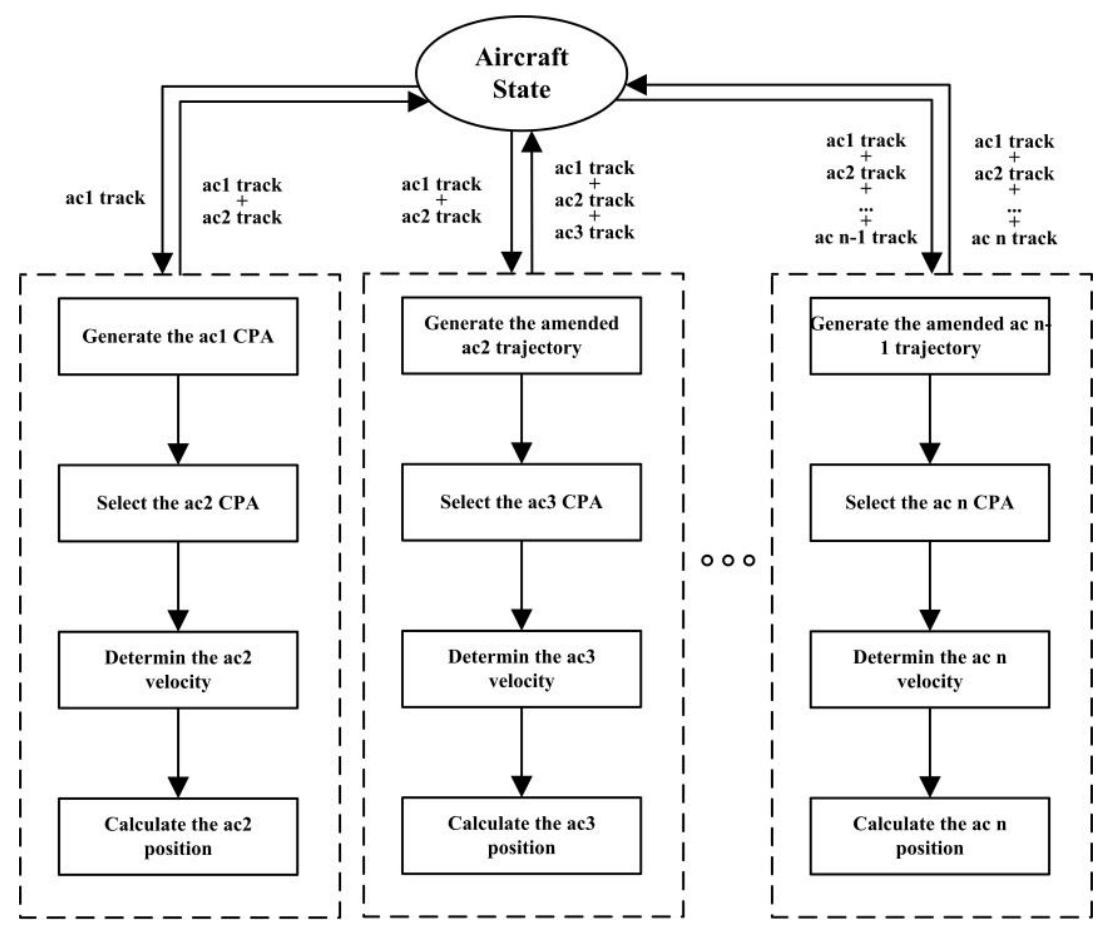

Figure 4. Scenario generation flow 


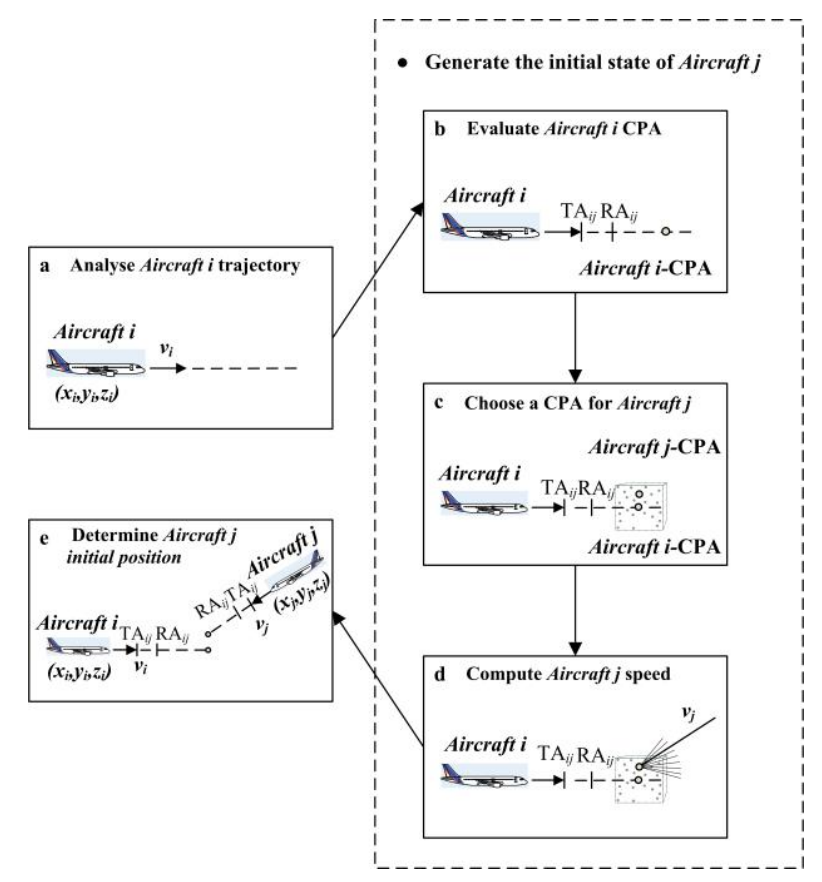

Figure 5. Conceptual depiction of aircraft state generation

Without loss of generality, Fig. 5 represents the sequence of tasks to compute the aircraft state variables (Aircraft j) based on the information of a known aircraft (Aircraft $i$ ) trajectory. The following computational process is used: Analyse Aircraft $i$ trajectory $\rightarrow$ Evaluate the Aircraft $i \mathrm{CPA} \rightarrow$ Choose a CPA geometry for Aircraft $j \rightarrow$ Compute the Aircraft $j$ speed $\rightarrow$ Determine Aircraft $j$ initial position. In Fig. 5 a, the state variables of Aircraft $i$ (speed, position, and time) are used as the inputs of the model. Suppose that there is a vehicle identified as Aircraft $j$ that has an encounter with Aircraft $i$. In Fig. $5 \mathrm{~b}$, based on the time criteria of TA and RA, a CPA for Aircraft $i$ is automatically computed by the model together with the CPA of Aircraft $j$. This CPA should be in the scope of the threat separation of Aircraft $i$ at $\mathrm{t}_{\mathrm{CPA}}$, as shown in In Fig. 5 c. In addition, the CPA of Aircraft $j$ is computed from a set of feasible geometries. Next, in Fig. 5 d, the possible 3D speed vectors of Aircraft $j$ that satisfy the performance constraint are computed again using the model. The speed vector of Aircraft $j$ may be variable over a certain range, leading to different possible future situations (state space) in which several potential collision scenarios could be induced. Based on the TA time criteria and the chosen horizontal and vertical speeds, the initial position of Aircraft $\mathrm{j}$ at time $\mathrm{t}$ is calculated in Fig. 5 e. Finally, the initial state of Aircraft $j$ is obtained as the output.

Note that in the calculation process, the TA and RA values are determined in the corresponding FL (DoT, 2011), while the CPA and speeds are uncertain but are within respective scopes. The different alternatives for Aircraft j's CPA and its possible speeds are used to generate the state space of scenarios. The optional values of speeds in the $\mathrm{x}, \mathrm{y}$ and $\mathrm{z}$ axes are bounded by a discrete finite domain; thus, the state space is not infinite. Obviously, it would be more realistic when the optional values are set with smaller intervals; however, using smaller intervals would result in the increase in the cost of computing time.

This research aims to enable the identification of all of the possible induced collision scenarios for the TCAS-equipped aircraft considering surrounding traffic. The initial conditions are easily parameterised considering only the specification of one aircraft's state and the amount of intruder aircraft because the states of the neighbouring aircraft are variable due to various sources of uncertainty. Based on the above step-by-step process, another state of neighbouring aircraft that would have a conflict with one of the existing aircraft could also be calculated.

When the scenario consists of 3 aircraft (one aircraft with a specified trajectory and two aircraft as the surrounding traffic), the process used for the generation of the initial state of Aircraft 3 is similar. At this time, the initial states of Aircraft 1 and Aircraft 2 serve as the inputs to compute the initial state of Aircraft 3 that is deemed to be the output. If Aircraft 3 has the potential for a collision with Aircraft 2, which is in the RA process of amending its trajectory to resolve the threat with Aircraft 1, then 
Aircraft 3 should be in the collision volume of Aircraft 2 in the RA process. Meanwhile, the possible position of Aircraft 3 at time $t$ is restricted to the threat volume of Aircraft 1. However, these aircraft are not approaching each other. Based on the CPA and the initial position, the speeds in the horizontal and vertical planes are calculated again by the model.

\subsection{Causal model based on TCAS II logic}

The proposed model must be initialised with an instance of a trajectory (Cartesian coordinates, speed and time) and the number of aircraft that should be considered as surrounding traffic in the hot spot scenario. The results generated by the model provide information to identify the potential induced collision scenarios based on step-by-step logic and also to explore the emergent dynamics (state space) between the resolution trajectories (RAs) and the neighbouring trajectories. The CPN model implements the description of the TCAS logic as a set of transitions. The model generates TCAS failures based on the logic sequence of activities presented in Fig. 4.

\subsubsection{Model representation}

For the implementation of a discrete model, the trajectory can be regarded as a sequence of 3D waypoints that the corresponding aircraft will follow, with the sequence containing the coordinates and speeds. The causal model that is developed for identifying the potential collisions between no more than 4 aircraft has been specified in the CPN formalism (Fig. 6, using 12 colours, 33 places and 13 transitions), and it mainly consists of three blocks of transitions that represent three different control events. These have similar functions that can be used to generate the initial state of the next neighbouring aircraft. Thus, as explained in Section 5.1, the model can be easily extended to generate induced collision scenarios that involve more aircraft by adding extra analogous blocks of transitions.

- Initialize the model with Aircraftl's initial state. In accordance with the actual situation and requirements, Aircraftl's initial state (3D position and speed, time) should be used as the input of the model to identify any future potential induced collisions.

- Generate the initial state of Aircraft 2 as a threat for Aircraft 1 (shown in Fig. 7). The first block contains three transitions (T1, T2, and T3), which specify the events of producing an encounter: T1 calculates the future CPA of Aircraft 1 based on the TA/RA time criteria, and the input of this block is the state information of Aircraft 1; T2 computes the CPA of Aircraft 2 that is in the minimum threat separation of Aircraft 1 at $t_{C P A}$. The different feasible CPAs of Aircraft 2 (the possible values are stored in Place "Variable1") contributes to the formation of state space. T3 assigns the optional speeds in 3D for Aircraft 2, and its initial position is determined based on the known speed and time. For example, the case tokens in Place "Vx1" are +1 ' $(0.17)+1$ ' $(0.15)$ which could be set as the initial speed of Aircraft 2 in x axis. The selected values from Places"Vx1" "Vy1" and "Vzl" form the 3D speed for Aircraft 2. Note that more extensive tokens are set in the actual simulation.

- Generate the initial state of Aircraft 3 as a threat/collision with Aircraft 2. The second block possesses five transitions (T4, T5, T6, T7, and T8): T4 copies the inputs of the initial states of Aircraft 1 and Aircraft 2 (one set of data for a potential collision and the other set for a possible threat); T5 obtains the start point of Aircraft 3, which is within the dimensions of the protected airspace of Aircraft 1 (not approaching) and the end point of Aircraft 3 that is in the collision volume of Aircraft 2 in its RA process; known start and end points, the speed of Aircraft 3 can be calculated by T6; T7 computes the CPA of Aircraft 3 that is within the minimum threat separation of Aircraft 1, which has amended its vertical speed; T8 assigns the optional speeds in 3D for Aircraft 3, and its initial position is determined based on the known speed and time.

- Generate the initial state of Aircraft 4, which has a threat/collision with Aircraft 3. The third block has five transitions (T9, T10, T11, T12, and T13), and their functions are similar to the corresponding transitions in the second block. 

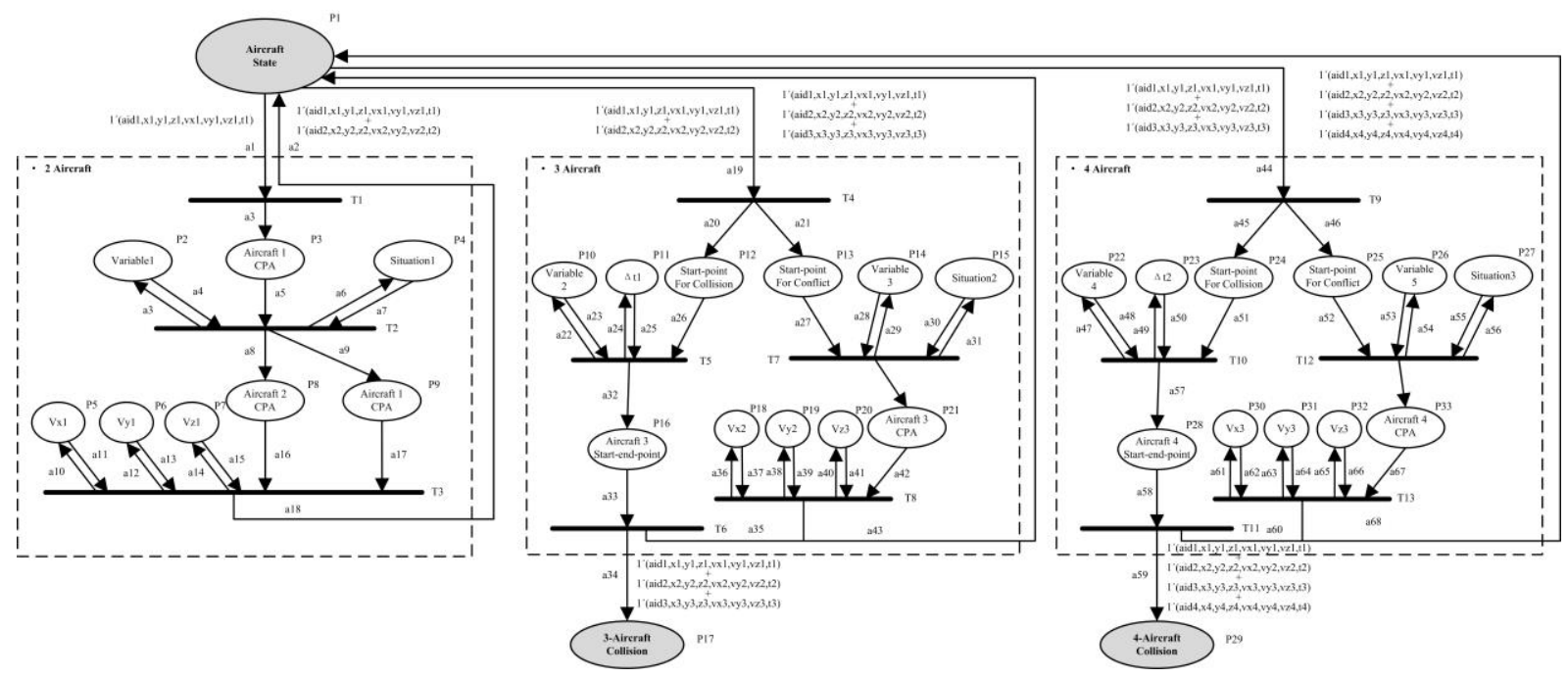

Figure 6. Causal model for TCAS logic

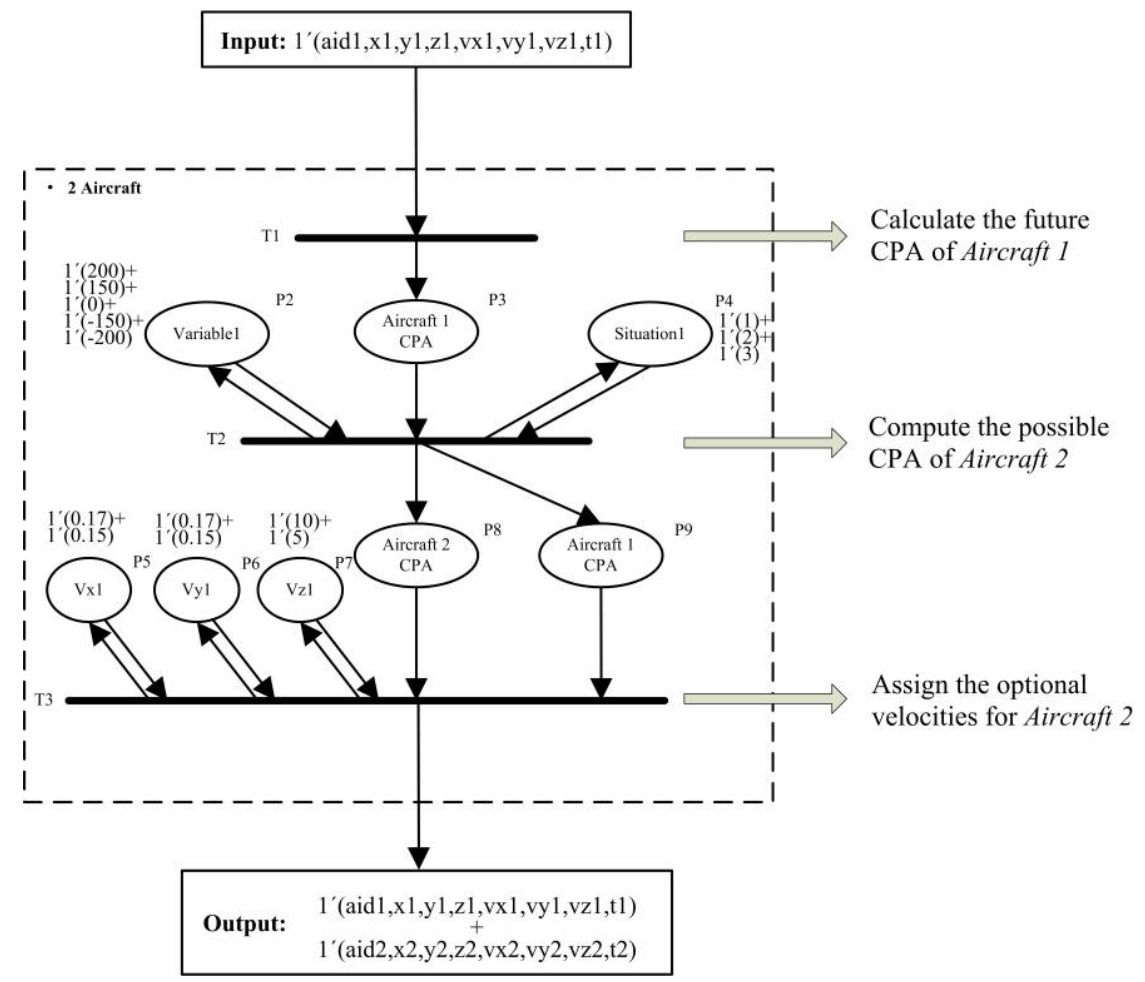

Figure 7. Flow chart of first block

\subsubsection{Net specification and description}

The discrete model considers situations that occur in a short period of time with a local scope. This results in a trajectory that can be approximated by a sequence of rectilinear timed segments of the aircraft (in a simplified view). The colours used to describe all of the information that are required in the relevant places are summarized in Table 2.

Table 2. Colour specification

\begin{tabular}{|c|c|c|}
\hline \multirow{2}{*}{ Colours } & \multicolumn{2}{c|}{ Description } \\
\cline { 2 - 3 } & Definition & Meaning \\
\hline aid & Int $1 \ldots \mathrm{N}$ & Aircraft identity \\
\hline
\end{tabular}




\begin{tabular}{|c|c|c|}
\hline$x$ & $\mathrm{R}$ & $x$ axis coordinate for 3D position \\
\hline$y$ & $\mathrm{R}$ & $y$ axis coordinate for 3D position \\
\hline$z$ & $\mathrm{R}$ & $z$ axis coordinate for 3D position \\
\hline$v x$ & $\mathrm{R}$ & Speed component in $x$ axis \\
\hline$v y$ & $\mathrm{R}$ & Speed component in $y$ axis \\
\hline$v z$ & $\mathrm{R}$ & Speed component in $z$ axis \\
\hline$d$ & $\mathrm{R}+$ & Vertical distance between aircraft at CPA \\
\hline$a l i m$ & $\mathrm{R}$ & Desired vertical minimum separation at CPA \\
\hline$t$ & Int $1 \ldots \mathrm{N}$ & Current time \\
\hline$\Delta t$ & Int $1 \ldots \mathrm{N}$ & Time interval \\
\hline$s$ & Int $1 \ldots \mathrm{N}$ & Optional situations \\
\hline
\end{tabular}

The specifications of the main places are shown in Table 3. Note that P22-P33 in the third block used to generate the initial state of Aircraft 4 have similar functions and characterizations as P10-P21 in the second block aiming to obtain the initial state of Aircraft 3. Therefore, only the operations of P1-P21 are detailed as follows.

Place "Aircraft State" contains tokens with eight colour attributes to define the initial position and speed information of an aircraft: aid is the ID of the corresponding aircraft; $(x, y, z)$ show the 3D coordinates; $(v x, v y, v z)$ indicate the 3D speed components; $t$ records the current time.

Places "Aircraft 1 CPA", "Aircraft 2 CPA", and "Aircraft 3 CPA" hold tokens with the same eight colour attributes as Place "Aircraft State" to keep the state information of the corresponding aircraft at the respective CPA.

Places "Vx1"/"Vx2", "Vy1"/"Vy2" and "Vz1"/"Vz2" separately hold several tokens with only one colour $v x / v y / v z$ as the constant options of the initial speed in each direction.

$\mathrm{P} 9$ is a copy of P3.

Places "Situation1" and "Situation2" own tokens with one colour $s$ as the identifier of possible threat situations.

Places "Variable1", "Variable2", and "Variable3" store tokens with the range of distance $d$ between each pair of aircraft.

Place "Start-point for Collision" memorizes the start-point information of the involved aircraft that would be used to generate an induced collision scenario. Place "Start-point for Threat" holds the remaining aircraft that would be used to generate a threat scenario. Place "Aircraft 3 Start-end-point" stores the calculated start and end points of Aircraft 3. Place "3-Aircraft Collision" preserves the position and speed information of the 3 aircraft, between which there would be a collision.

Table 3. Place specification

\begin{tabular}{|c|c|c|c|}
\hline \multirow{2}{*}{ Num. } & \multirow{2}{*}{ Places } & \multicolumn{2}{|r|}{ Description } \\
\hline & & Colour & Definition \\
\hline $\mathrm{P} 1$ & Aircraft State & AS & $\operatorname{aid}^{*} x * y^{*} z * v x^{*} v y^{*} v z^{*} t$ \\
\hline $\mathrm{P} 2$ & Variable1 & $\mathrm{V} 1$ & $d$ \\
\hline P3 & Aircraft 1 CPA & $\mathrm{AC1}$ & $\operatorname{aid}^{*} x * y^{*} z^{*} v x^{*} v y * v z^{*} t$ \\
\hline $\mathrm{P} 4$ & Situation1 & S1 & $s$ \\
\hline P5 & Vx1 & $\mathrm{X} 1$ & $v x$ \\
\hline P6 & Vy1 & Y1 & vy \\
\hline P7 & Vz1 & $\mathrm{Z1}$ & $v z$ \\
\hline P8 & Aircraft 2 CPA & $\mathrm{AC} 2$ & $\operatorname{aid}^{*} x * y^{*} z * v x * v y * v z^{*} t$ \\
\hline P9 & Aircraft 1 CPA & $\mathrm{AC} 1$ & $\operatorname{aid}^{*} x^{*} y^{*} z^{*} v x^{*} v y^{*} v z^{*} t$ \\
\hline $\mathrm{P} 10$ & Variable2 & $\mathrm{V} 2$ & $d$ \\
\hline P11 & $\Delta \mathrm{t} 1$ & $\mathrm{~T}$ & $\Delta t$ \\
\hline $\mathrm{P} 12$ & Start-point for Collision & SP1 & $a i d * x * y * z * v x * v y * v z * t$ \\
\hline $\mathrm{P} 13$ & Start-point for Threat & SP2 & $\operatorname{aid}^{*} x^{*} y^{*} z^{*} v x^{*} v y^{*} v z^{*} t$ \\
\hline P14 & Variable3 & V3 & $d$ \\
\hline $\mathrm{P} 15$ & Situation2 & $\mathrm{S} 2$ & $s$ \\
\hline P16 & Aircraft 3Start-end-point & ASE & $\operatorname{aid}^{*} x * y^{*} z * v x * v y * v z^{*} t$ \\
\hline $\mathrm{P} 17$ & 3-Aircraft Collision & $3 \mathrm{AC}$ & $\operatorname{aid}^{*} x^{*} y^{*} z^{*} v x^{*} v y^{*} v z^{*} t$ \\
\hline P18 & $\mathrm{Vx} 2$ & $\mathrm{X} 2$ & \\
\hline
\end{tabular}




\begin{tabular}{|c|c|c|c|}
\hline P19 & Vy2 & Y2 & $v y$ \\
\hline P20 & Vz2 & Z2 & $v z$ \\
\hline P21 & Aircraft 3 CPA & AC3 & aid $x^{*} y^{*} z^{*} v x^{*} v y^{*} v z^{*} t$ \\
\hline
\end{tabular}

\section{Results}

Table 4 provides the data used in the different experiments that are used to illustrate the feasibility of the CPN model.

Table 4. Parameter values for the scenarios

\begin{tabular}{|c|c|c|c|c|c|c|}
\hline Equipment & $\begin{array}{c}\text { Detection } \\
\text { range } \\
\text { (NM) }\end{array}$ & $\begin{array}{c}\text { RA } \\
\text { acceleration } \\
(\mathbf{g})\end{array}$ & $\begin{array}{c}\text { primary/strengthening } \\
\text { RA Pilot Delay } \\
(\mathbf{s})\end{array}$ & $\begin{array}{c}\text { Horizontal } \\
\text { size } \\
(\mathbf{m})\end{array}$ & $\begin{array}{c}\text { Vertical } \\
\text { size } \\
(\mathbf{m})\end{array}$ & $\begin{array}{c}\text { SL } \\
\text { TCAS II } \\
7.1\end{array}$ \\
40 & $0.25 /-0.25$ & $5 / 3$ & 40.74 & 11.95 & 6 \\
\hline
\end{tabular}

The diameter and height of the collision cylinders are twice as long as the horizontal and vertical sizes of the aircraft, respectively (i.e., $\mathrm{D}_{\mathrm{cl}}=0.044 \mathrm{NM}$ and $\mathrm{H}_{\mathrm{cl}}=78.44 \mathrm{ft}$ ) (EuroControl, 2001). The computer used for this simulation is an EliteBook laptop with a $2.6 \mathrm{GHz}$ Intel i5 processor and $4 \mathrm{~GB}$ of RAM, which is enough for the memory requirements of the algorithmic operations and simulation.

\subsection{Three-aircraft scenarios}

In this section, the results from threat resolution of one-on-one encounter with only one aircraft (from the surrounding traffic) are summarized. The different induced collision scenarios have been grouped into four different scenarios.

\subsubsection{Case representation}

The three-aircraft scenario is shown in Fig. 2 and depicted in Section 2. The three aircraft are all in the sixth SL. At 15:22:56, Aircraft 1 is cruising at (8.15 NM, 3.69 NM) in FL 145 with a ground speed of $461 \mathrm{kt}$; Aircraft 2 is at $(20.90 \mathrm{NM}, 3.69 \mathrm{NM})$ with a ground speed of $612 \mathrm{kt}$ and descends from FL160 with a vertical speed of $100.2 \mathrm{fpm}$; Aircraft 3 is at $(2.91 \mathrm{NM}, 3.67 \mathrm{NM})$ with a ground speed of $652 \mathrm{kt}$ and climbs slightly from FL148 with a vertical speed of $120.0 \mathrm{fpm}$.

For resolving the threat between Aircraft 1 and Aircraft 2, the TA is to fire a warning at 15:23:01. As the situation is getting worse, a RA emerges 15 seconds later to ask the crew to climb/descend for this encounter (15:23:16). At first, Aircraft 3 does not experience a threat with Aircraft 2. However, they encounter each other as a result of the amended flight level of Aircraft 2. Table 5 illustrates the waypoints of a partial trajectory of each aircraft before the collision occurs. At 15:23:32, Aircraft 2 and Aircraft 3 burst into each other's collision volumes, their horizontal distance is $\sqrt{(15.50-15.51)^{2}+(6.57-6.55)^{2}}=0.022 \mathrm{Nm}<0.044 \mathrm{Nm}$, and the altitude interval is $14880-14825=55 f t<78.44 \mathrm{ft}$. The induced threat between Aircraft 2 and Aircraft 3 is detected at 15:23:28, and the pilot action time is set as 5 seconds. Thus, the encounter would degenerate into a collision.

Table 5. Waypoints of partial trajectory

\begin{tabular}{|c|c|c|c|c|c|c|c|c|c|}
\hline Time & Aircraft & $\mathbf{X}(\mathbf{N M})$ & $\mathbf{Y}(\mathbf{N M})$ & $\mathbf{Z}(\mathbf{f t})$ & Time & Aircraft & $\mathbf{X}(\mathbf{N M})$ & $\mathbf{Y}(\mathbf{N M})$ & $\mathbf{Z}(\mathbf{f t})$ \\
\hline 15:23:17 & Aircraft 1 & 10.25 & 5.37 & 14500.00 & $15: 23: 25$ & Aircraft 1 & 11.05 & 6.01 & 14573.33 \\
\hline 15:23:17 & Aircraft 2 & 17.75 & 5.37 & 14850.00 & $15: 23: 25$ & Aircraft 2 & 16.55 & 6.01 & 14836.67 \\
\hline 15:23:17 & Aircraft 3 & 10.26 & 5.35 & 14850.00 & $15: 23: 25$ & Aircraft 3 & 13.06 & 5.99 & 14866.00 \\
\hline 15:23:18 & Aircraft 1 & 10.35 & 5.45 & 14596.67 & $15: 23: 26$ & Aircraft 1 & 11.15 & 6.09 & 14570.00 \\
\hline 15:23:18 & Aircraft 2 & 17.60 & 5.45 & 14848.33 & $15: 23: 26$ & Aircraft 2 & 16.40 & 6.09 & 14835.00 \\
\hline 15:23:18 & Aircraft 3 & 10.61 & 5.43 & 14852.00 & $15: 23: 26$ & Aircraft 3 & 13.41 & 6.07 & 14868.00 \\
\hline 15:23:19 & Aircraft 1 & 10.45 & 5.53 & 14593.33 & $15: 23: 27$ & Aircraft 1 & 11.25 & 6.17 & 14566.67 \\
\hline
\end{tabular}




\begin{tabular}{|l|l|l|l|l|l|l|l|l|l|}
\hline 15:23:19 & Aircraft 2 & 17.45 & 5.53 & 14846.67 & $15: 23: 27$ & Aircraft 2 & 16.25 & 6.17 & 14833.33 \\
\hline 15:23:19 & Aircraft 3 & 10.96 & 5.51 & 14854.00 & $15: 23: 27$ & Aircraft 3 & 13.76 & 6.15 & 14870.00 \\
\hline 15:23:20 & Aircraft 1 & 10.55 & 5.61 & 14590.00 & $15: 23: 28$ & Aircraft 1 & 11.35 & 6.25 & 14563.33 \\
\hline $15: 23: 20$ & Aircraft 2 & 17.30 & 5.61 & 14845.00 & $15: 23: 28$ & Aircraft 2 & 16.10 & 6.25 & 14731.67 \\
\hline $15: 23: 20$ & Aircraft 3 & 11.31 & 5.59 & 14856.00 & $15: 23: 28$ & Aircraft 3 & 14.11 & 6.23 & 14872.00 \\
\hline $15: 23: 21$ & Aircraft 1 & 10.65 & 5.69 & 14586.67 & $15: 23: 29$ & Aircraft 1 & 11.45 & 6.33 & 14560.00 \\
\hline 15:23:21 & Aircraft 2 & 17.15 & 5.69 & 14843.33 & $15: 23: 29$ & Aircraft 2 & 15.95 & 6.33 & 14830.00 \\
\hline 15:23:21 & Aircraft 3 & 11.66 & 5.67 & 14858.00 & $15: 23: 29$ & Aircraft 3 & 14.46 & 6.31 & 14874.00 \\
\hline $15: 23: 22$ & Aircraft 1 & 10.75 & 5.77 & 14583.33 & $15: 23: 30$ & Aircraft 1 & 11.55 & 6.41 & 14556.67 \\
\hline $15: 23: 22$ & Aircraft 2 & 17.00 & 5.77 & 14841.67 & $15: 23: 30$ & Aircraft 2 & 15.80 & 6.41 & 14828.33 \\
\hline 15:23:22 & Aircraft 3 & 12.01 & 5.75 & 14860.00 & $15: 23: 30$ & Aircraft 3 & 14.81 & 6.39 & 14876.00 \\
\hline $15: 23: 23$ & Aircraft 1 & 10.85 & 5.85 & 14580.00 & $15: 23: 31$ & Aircraft 1 & 11.65 & 6.49 & 14553.33 \\
\hline $15: 23: 23$ & Aircraft 2 & 16.85 & 5.85 & 14840.00 & $15: 23: 31$ & Aircraft 2 & 15.65 & 6.49 & 14826.67 \\
\hline $15: 23: 23$ & Aircraft 3 & 12.36 & 5.83 & 14862.00 & $15: 23: 31$ & Aircraft 3 & 15.16 & 6.47 & 14878.00 \\
\hline $15: 23: 24$ & Aircraft 1 & 10.95 & 5.93 & 14576.67 & $15: 23: 32$ & Aircraft 1 & 11.75 & 6.57 & 14550.00 \\
\hline $15: 23: 24$ & Aircraft 2 & 16.70 & 5.93 & 14838.33 & $15: 23: 32$ & Aircraft 2 & 15.50 & 6.57 & 14825.00 \\
\hline $15: 23: 24$ & Aircraft 3 & 12.71 & 5.91 & 14864.00 & $15: 23: 32$ & Aircraft 3 & 15.51 & 6.55 & 14880.00 \\
\hline
\end{tabular}

\subsubsection{Collision induced scenarios with three aircraft}

Based on the scenario generation process of the causal model, first, the state of the second aircraft (without a loss of generality, it is called Aircraft 2) that has an encounter with known Aircraft 1 is calculated. Then, the state of the third aircraft (Aircraft 3) that is restricted to the threat volume of Aircraft 1 (not approaching) and would have a collision with Aircraft 2 flying in the amended trajectory can be computed. Thus, for the generation of 3-aircraft collision scenarios can be simply understood as:

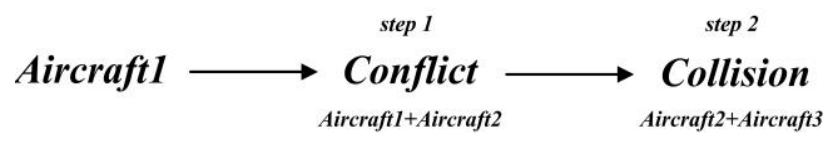

Based on the analysis of the results generated by the causal model, all of the collision scenarios can be classified into four typical situations that are summarized in Table 6 . The differences between these are the relative CPA positions of Aircraft 1 and Aircraft 2 involved in the encounter and the initial state of Aircraft 2 (climb/descend). However, no matter whether Aircraft 2 climbs or descends, the RA direction is selected in view of the CPA position (as shown in Table 6, case 3 is similar to case 1 and case 4 is similar to case 2). Thus, the four collision scenarios of three aircraft can be merged into two typical scenarios (case 1 and case 2). These mainly consider the relative CPA altitude (directly affecting the change in RA).

Table 6. Typical collision scenarios of three aircraft

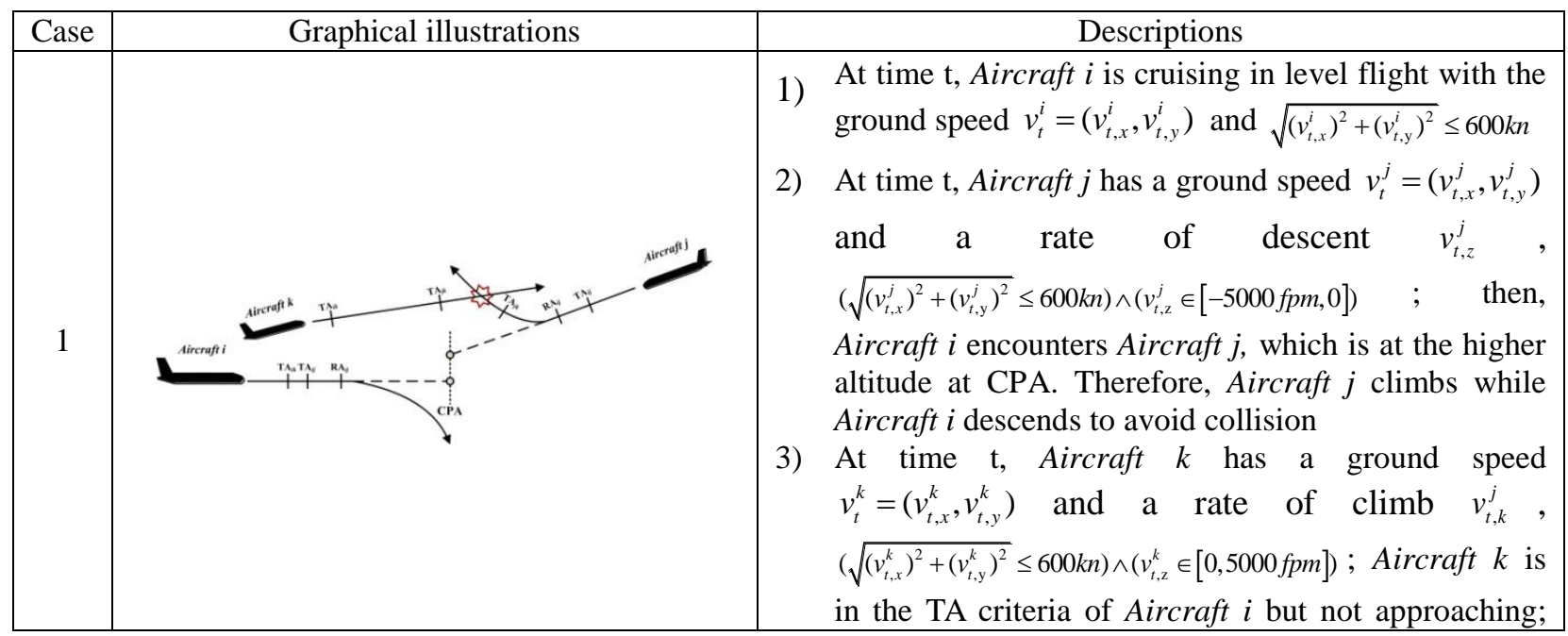




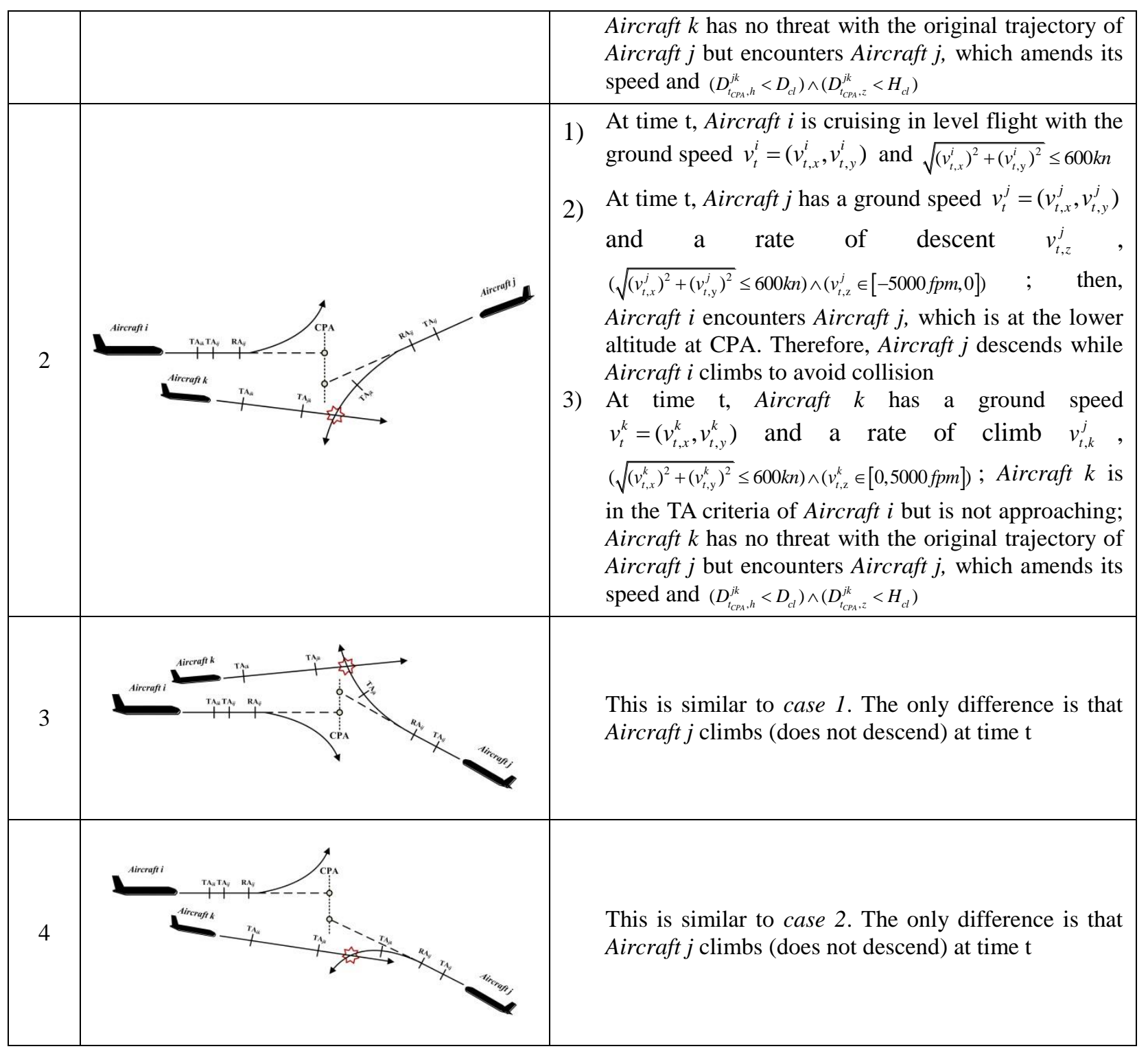

\subsection{Four-aircraft scenarios}

This section summarizes the results from threat resolutions (RAs) of one-on-one encounters with two aircraft in the surrounding traffic. The different induced collision scenarios have been grouped into eight different scenarios.

\subsubsection{Case representation}

To illustrate the possibility of induced collisions between four aircraft, let us consider the traffic scenario illustrated in Fig. 8. In this scenario, four fully equipped aircraft are considered with two predicted encounters (threat 1 between Aircraft 1 and Aircraft 2, and the other one is threat 2 between Aircraft 3 and Aircraft 4) (Tang et al., 2014). Variable $t_{T A}^{i}(i=1,2,3,4)$ is used for the TA emergence time, and variable $t_{R A}^{i}(i=1,2,3,4)$ indicates the RA. In normal flight, Aircraft 1 is cruising at FL155 and Aircraft 2 is cruising at FL160 on an opposing route. When Aircraft 2 starts a descending operation and flies into the range of Aircraft 1, a TA is issued by TCAS to warn the crew of Aircraft 1 that a collision is predicted to occur within $t_{T A}^{1}$. An RA is issued at $t_{R A}^{1}$ to provide a suggestion to the pilot. Once the threat is detected, Aircraft 1 performs a descend operation while Aircraft 2 climbs to provide the greatest vertical separation at CPA. Normally, the RA strength selects the ALIM as the smallest safe separation that requires a minimal speed change. Meanwhile, a similar TA and RA process is initiated 
between Aircraft 3 and Aircraft 4. When Aircraft 4 comes within the range of Aircraft 3 and a collision is predicted to occur, a TA is issued at $t_{T A}^{3}$ and an RA is issued at $t_{R A}^{3}$. The crew in Aircraft 3 respond to the RA by attempting to descend, while Aircraft 4 climbs with the strength of ALIM. Unfortunately, despite the RA's resolution of both encounters, a new secondary threat is initiated between Aircraft 4 and Aircraft 1 as a consequence of previous decisions. This is detected by the TCAS at 20:45:24. The crew has to address the emergent encounter. However, there is not enough time left for the pilot to avoid this collision.

In the future high-density traffic with the wide use of RPAS, these situations are more likely to appear. For a pair of aircraft which are in the RA process, if another aircraft originally cruises in the neighbouring FL and speeds up, a subsequent new RA would be promptly issued and the dangerous situation that may initiate a collision could emerge.

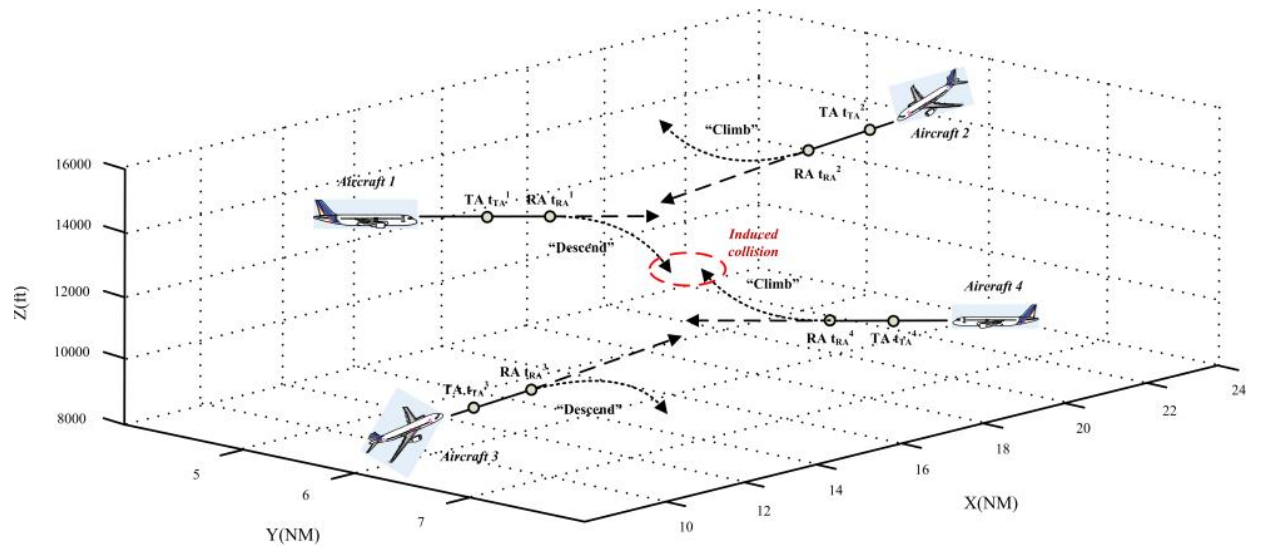

Figure 8. Four-aircraft scenario

The four aircraft are all in the sixth SL. At 20:44:56, Aircraft 1 is cruising at (10.75 NM, 3.56 $\mathrm{NM})$ in FL 155 with a ground speed of $461 \mathrm{kt}$; Aircraft 2 is at $(20.50 \mathrm{NM}, 3.56 \mathrm{NM})$ with a ground speed of $612 \mathrm{kt}$ and descends from FL160 with a vertical speed of $100.2 \mathrm{fpm}$; Aircraft 3 is at (2.49 $\mathrm{NM}, 3.45 \mathrm{NM}$ ) with a ground speed of $469 \mathrm{kt}$ and climbs slightly from FL148 with a vertical speed of $180.0 \mathrm{fpm}$. Aircraft 4 is cruising at $(21.98 \mathrm{NM}, 9.35 \mathrm{NM})$ with the height of $15455 \mathrm{ft}$ and its ground speed is $672 \mathrm{kt}$.

For threat 1, when Aircraft 1 and Aircraft 2 fly within range of one another, a TA is issued to fire a warning at 20:45:01. However, the situation is getting worse, and 15 seconds later a RA emerges to ask the crew to act upon this encounter (20:45:16). For threat 2, the TA is issued at 20:45:05, and the subsequent RA appears when the RA criteria are met (20:45:20). Table 7 illustrates the waypoints of a partial trajectory of each aircraft before the collision occurs. At 20:45:28, Aircraft 1 and Aircraft 4 burst into each other's collision safety volume, their horizontal distance is $\sqrt{(13.95-13.98)^{2}+(6.12-6.15)^{2}}=0.042 \mathrm{Nm}<0.044 \mathrm{Nm}$, and the altitude interval is $15476.67-15470.00=76.67 f t<78.44 f t$.

Table 7. Waypoints of partial trajectory

\begin{tabular}{|c|c|c|c|c|c|c|c|c|c|}
\hline Time & Aircraft & $\mathbf{X}(\mathbf{N M})$ & $\mathbf{Y ( N M )}$ & $\mathbf{Z}(\mathbf{f t})$ & Time & Aircraft & $\mathbf{X ( N M )}$ & $\mathbf{Y ( N M )}$ & $\mathbf{Z}(\mathbf{f t})$ \\
\hline 20:45:21 & Aircraft 1 & 13.25 & 5.56 & 15500.00 & $20: 45: 25$ & Aircraft 1 & 13.65 & 5.88 & 15486.67 \\
\hline 20:45:21 & Aircraft 2 & 20.75 & 5.56 & 15850.00 & $20: 45: 25$ & Aircraft 2 & 20.15 & 5.88 & 15843.33 \\
\hline $20: 45: 21$ & Aircraft 3 & 6.24 & 3.45 & 15365.00 & $20: 45: 25$ & Aircraft 3 & 6.84 & 3.45 & 15377.00 \\
\hline $20: 45: 21$ & Aircraft 4 & 15.73 & 6.85 & 15455.00 & $20: 45: 25$ & Aircraft 4 & 14.73 & 6.45 & 15455.00 \\
\hline 20:45:22 & Aircraft 1 & 13.35 & 5.64 & 15496.67 & $20: 45: 26$ & Aircraft 1 & 13.75 & 5.96 & 15483.33 \\
\hline 20:45:22 & Aircraft 2 & 20.60 & 5.64 & 15848.33 & $20: 45: 26$ & Aircraft 2 & 20.00 & 5.96 & 15841.67 \\
\hline $20: 45: 22$ & Aircraft 3 & 6.39 & 3.45 & 15368.00 & $20: 45: 26$ & Aircraft 3 & 6.99 & 3.45 & 15375.00 \\
\hline 20:45:22 & Aircraft 4 & 15.48 & 6.75 & 15455.00 & $20: 45: 26$ & Aircraft 4 & 14.48 & 6.35 & 15460.00 \\
\hline 20:45:23 & Aircraft 1 & 13.45 & 5.72 & 15493.33 & $20: 45: 27$ & Aircraft 1 & 13.85 & 6.04 & 15480.00 \\
\hline 20:45:23 & Aircraft 2 & 20.45 & 5.72 & 15846.67 & $20: 45: 27$ & Aircraft 2 & 19.85 & 6.04 & 15840.00 \\
\hline
\end{tabular}




\begin{tabular}{|l|l|c|c|c|c|c|c|c|c|}
\hline 20:45:23 & Aircraft 3 & 6.54 & 3.45 & 15371.00 & $20: 45: 27$ & Aircraft 3 & 7.14 & 3.45 & 15373.00 \\
\hline $20: 45: 23$ & Aircraft 4 & 15.23 & 6.65 & 15455.00 & $20: 45: 27$ & Aircraft 4 & 14.23 & 6.25 & 15465.00 \\
\hline $20: 45: 24$ & Aircraft 1 & 13.55 & 5.80 & 15490.00 & $20: 45: 28$ & Aircraft 1 & 13.95 & 6.12 & 15476.67 \\
\hline $20: 45: 24$ & Aircraft 2 & 20.30 & 5.80 & 15845.00 & $20: 45: 28$ & Aircraft 2 & 19.70 & 6.12 & 15838.33 \\
\hline $20: 45: 24$ & Aircraft 3 & 6.69 & 3.45 & 15374.00 & $20: 45: 28$ & Aircraft 3 & 7.29 & 3.45 & 15371.00 \\
\hline 20:45:24 & Aircraft 4 & 14.98 & 6.55 & 15455.00 & $20: 45: 28$ & Aircraft 4 & 13.98 & 6.15 & 15470.00 \\
\hline
\end{tabular}

\subsubsection{Collision induced scenarios with four aircraft}

Based on the scenario generation process of the causal model, first, the state of the second aircraft (called Aircraft 2, without a loss of generality) that has an encounter with known Aircraft 1 is calculated. Then, the state of the third aircraft (Aircraft 3) that would have a collision or threat with Aircraft 2 is computed. If this is a collision between Aircraft 2 and Aircraft 3 (e.g., the above case), then the fourth aircraft (Aircraft 4) can be assumed to have an encounter with Aircraft 3. If it is a threat between Aircraft 2 and Aircraft 3, then the fourth aircraft (Aircraft 4) can be assumed to have a collision with Aircraft 3. The generation of 4-aircraft collision scenarios can be simply understood as:

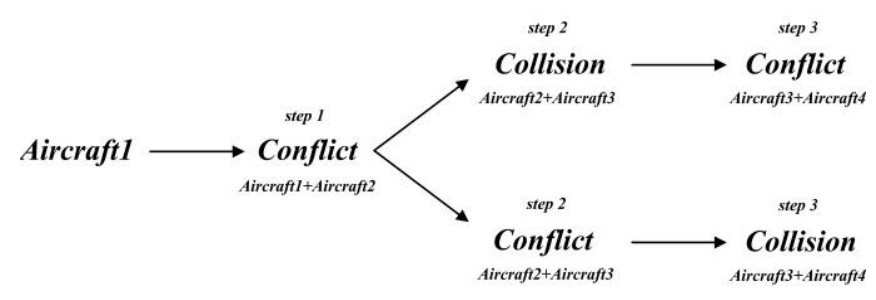

Based on analysis of the results generated by the causal model, all of the collision scenarios could be classified into eight typical situations. These are summarized in Table 8. For case 1, the scenario could be seen as two separate but interactional threats that initiate the collision between two aircraft in their own RA process. Altering the relative CPA altitudes of the aircraft that are involved in each threat would give rise to four typical collision scenarios. This is explained in Table 8, and these correspond to the condition that step 2 is a collision. For case 5, Aircraft 2 first has a threat with Aircraft 1, and then it climbs but encounters Aircraft 3. Here, the two sequent threats are not separate, and there are also four typical collision scenarios illustrated in Table 8. These correspond to the condition that step 2 is threat and the collision is generated in step 3. The relationships between the four aircraft appear to be in a loop that could be called a "deadlock".

Table 8. Typical collision scenarios of four-aircraft

\begin{tabular}{|c|c|c|c|}
\hline Case & Graphical illustrations & & Descriptions \\
\hline 1 & & & 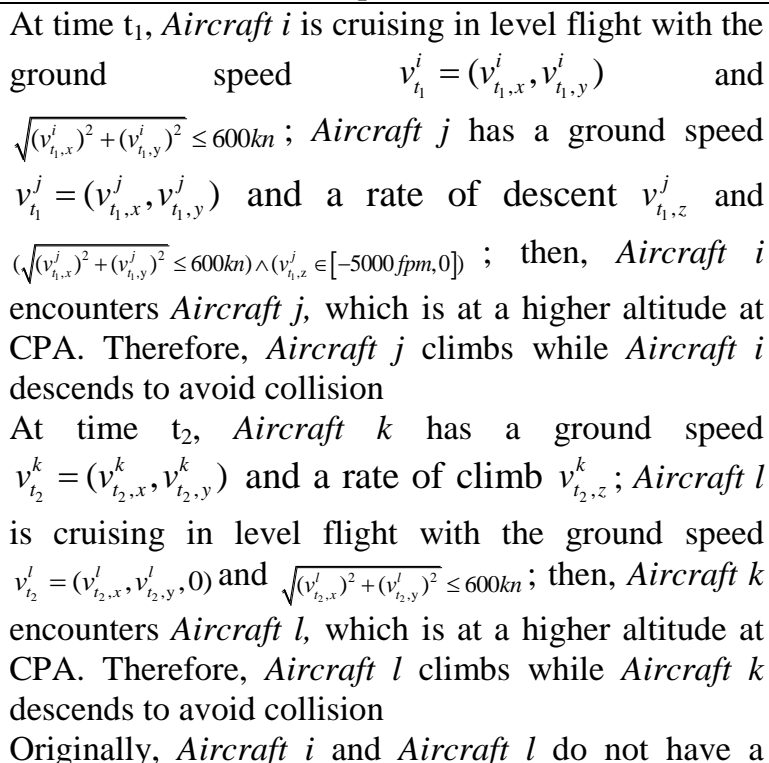 \\
\hline
\end{tabular}




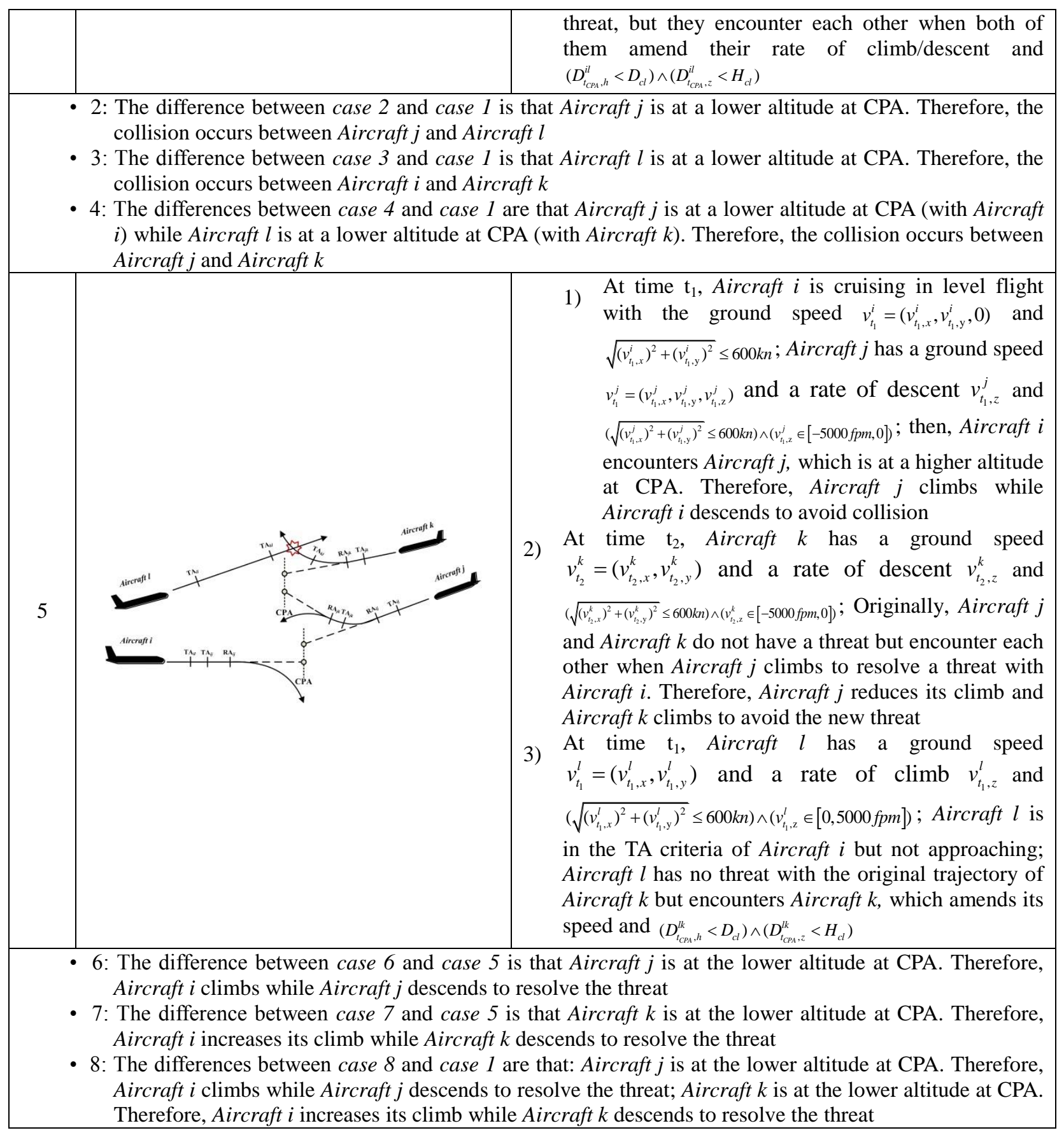

\subsection{Induced collision scenarios}

Based on the scenario generation process, the causal model can be extended to determine all of the collision scenarios for a given aircraft trajectory and a particular amount of aircraft as surrounding traffic. The initial states of the multiple aircraft that are involved in the scenarios are generated one by one. The unique constraint is the rapid expansion of the state space.

In addition, according to Billingsley et al. (2009), induced encounter scenarios with only three aircraft realistically comprise over $95 \%$ of the cases (based on analysing the distribution of the number of aircraft involved in such induced encounter situations). There was only one situation that involved seven aircraft (based on examining 3803 induced encounter situations that were identified using radar data from the FAA and Department of Defense sites throughout the United States). Therefore, the causal model was extended to generate the state space of different numbers of aircraft involved in a local situation. The results are illustrated in Table 9. 
Table 9. Summarized results of multi-aircraft scenarios

\begin{tabular}{|c|c|c|}
\hline $\begin{array}{c}\text { Aircraft } \\
\text { number }\end{array}$ & $\begin{array}{c}\text { Typical induced } \\
\text { collision scenarios }\end{array}$ & $\begin{array}{c}\text { Computation } \\
\text { Time }\end{array}$ \\
\hline 3 & 2 & $34^{\prime}$ \\
\hline 4 & 8 & $2^{\prime} 21^{\prime \prime}$ \\
\hline 5 & 24 & $15^{\prime} 52^{\prime \prime}$ \\
\hline 6 & 64 & $1: 32^{\prime} 47^{\prime \prime}$ \\
\hline 7 & 160 & $4: 56^{\prime} 38^{\prime \prime}$ \\
\hline
\end{tabular}

Analysing the typical collision scenarios is a Non-deterministic Polynomial (NP) problem that cannot be solved by exploring all of the combinations. In reality, there should be a saturation of the density that can be accepted in a local region. Therefore, there is no need to create a fully specified induced encounter model designed for the collision scenario generation of many aircraft in a local airspace. A fully specified causal encounter model with four aircraft (shown in Section 5.2) would require at least 12 colours, 33 places and 13 transitions. More aircraft would need more parameters. Based on the summarized results (typical collision scenarios and computation time), each additional aircraft increases the number of potential variable correlations by an exponential amount. Therefore, this significantly raises the possible collision scenarios (state space). Furthermore, induced encounter scenarios in real airspace, as already noted, are relatively rare.

\section{Conclusions}

TCAS is designed to provide last-resort CA guidance directly to pilots. Long-term operational experience has demonstrated the utility and efficiency of TCAS. However, it is widely accepted that a collision also could occur in an exceptional situation, even if all of the involved aircraft are TCASequipped, e.g., the Überlingen mid-air collision in 2002 (Johnson, 2004). TCAS can actually induce a collision that would not have otherwise occurred, especially with dense surrounding traffic, when multiple aircraft are involved. There is a lack of causal models in the public domain to characterise the surrounding traffic scenarios that could initiate an induced collision; such scenarios could be used for the comparison of those actual traffic scenarios to reduce collision frequency. The motivation for the development of this causal model is to identify TCAS induced collisions and to support follow-up research for the safety analysis of current and advanced ATM concepts including TCAS. The main contributions of this paper are as follows:

- A mathematical model for the TCAS II algorithm was developed to be potentially used in a TCAS-TCAS encounter. TCAS II provides TAs to warn pilots of the encounter with neighbouring traffic and RAs to prevent a collision by offering a suggested resolution manoeuvre to pilots to execute an avoidance manoeuvre in the vertical direction. Using a series of mathematical equations, this CA process has been conceptually described. The process has also been used as the theoretical basis for construction of the encounter model.

- A novel scenario generation process of potential collisions was proposed. The only input of this model is the aircraft trajectory and the number of intruder aircraft, while the inputs of most other encounter models (Netjasov et al., 2013; Kochenderfer et al., 2010; Billingsley et al.,2009; Tang et al., 2014) are the initial states (e.g., trajectories) of all involved aircraft for the analysis of particular traffic geometries. In contrast, the proposed model presented in this paper aims to generate the potential collision scenarios for a certain number of aircraft, based on the trajectory of just one instance representative aircraft, rather than to test whether a multi-aircraft scenario contains a potential collision or not. The generated encounter scenarios may not be credible within the normal operation of the ATC system, as some of them are designed through the use of different cruising flight levels.

- The encounter model is represented in the CPN formalism. This causal model depicts the procedure that takes an aircraft with a known initial state into various induced collision scenarios containing multiple TCAS-equipped aircraft. With the state space, the model provides a better understanding of the potential collision occurrences for risk assessment by comprehending the cause-effect relationship of each action. The initial states of multiple 
aircraft that are involved in the scenarios can be generated one by one. Finally, all of the possible situations (state space) can be represented for subsequent analysis to summarise the typical induced collision scenarios.

- A summary is provided of the typical induced collision scenarios based on the simulation results of the causal model. Quantitative measurement experiments were conducted to validate the feasibility and effectiveness of the encounter model. In addition, for scenarios involving three or four aircraft (representative of almost all of the factual situations), the detailed process of a collision and the typical scenarios were illustrated and described in detail.

The generated state space of all possible induced collision scenarios could be stored in a database, and an advanced warning could be automatically displayed when the traffic in a particular airspace volume matches one of the scenarios identified by this model. In addition, to accomplish the fundamental purpose of our research, the next steps are 1) to develop the pattern recognition tool to recognise those traffic scenarios in which a potential induced collision may exist and 2) to utilise the proposed causal model to promote the improvement of the current TCAS logic, which is intended to address the future hectic and congested traffic with the enhanced CA performance.

\section{Acknowledgment}

This research is partially supported by the Ministry of Economy and Competitiveness in the project "Fire Guided Unmanned Aircrafts and Resources Distribution-2 (Fire GUARD)", MINECO Spanish program TIN2014-56919-C3-1-R; H2020 "Adaptive self-Governed aerial Ecosystem by Negotiated Traffic" (AGENT); National Natural Science Foundation of China, 61202336.

\section{References}

Billingsley, T.B., Espindle, L.P., Griffith, J.D., 2009. TCAS multiple threat encounter analysis. MIT Lincoln Laboratory, Project Report ATC-359, 2009.

Billingsley, T.B., Kochenderfer, M.J., Chryssanthacopoulos, J.P., 2012. Collision avoidance for general aviation. IEEE Aerospace and Electronic Systems Magazine 27(7), 4-12.

Brooker, P., 2013. Introducing unmanned aircraft systems into a high reliability ATC system. Journal of Navigation 66(5), 719-735.

Carvalho, P.V.R., Gomes, J.O., Huber, G.J., Vidal, M.C., 2009. Normal people working in normal organizations with normal equipment: System safety and cognition in a mid-air collision. Applied ergonomics 40(3), 325-340.

Catena, A., Melita, C.D., Muscato, G., 2014. Automatic tuning architecture for the navigation control loops of unmanned aerial vehicles. Journal of Intelligent \& Robotic Systems 73(1-4), 413-427.

Chryssanthacopoulos, J.P., Kochenderfer, M.J., 2011. Collision avoidance system optimization with probabilistic pilot response models. American Control Conference (ACC). San Francisco, CA, 29 June-1 July, 2011, pp. 2765-2770.

Conde, R., Alejo, D., Cobano, J.A., Viguria, A., Ollero, A., 2012. Conflict detection and resolution method for cooperating unmanned aerial vehicles. Journal of Intelligent \& Robotic Systems 65(1-4): 495-505.

Department of Trade, 1982. Aircraft Accident Report 9/82, Report on the Collision in the Zagreb Area, Yugoslavia, on 10 September 1976, Accident Investigation Branch, UK.

DoT, 2011. Introduction to TCAS II Version 7.1. Department of Transportation, Federal Aviation Administration, USA.

Espindle, L.P., Griffith, J.D., Kuchar, J.K., 2009. Safety analysis of upgrading to TCAS version 7.1 using the 2008 US Correlated Encounter Model. Project Report ATC-349, Lincoln Laboratory.

EuroControl, 2001. The EUR RVSM pre-implementation safety case, Version 2.0. Document RVSM 691.

Garcia-Chico, J.L., Corker, K.M., 2007. An analysis of operational errors and the interaction with TCAS RA's. In: Proceedings 7th USA/Europe Air Traffic Management R\&D Seminar (ATM2007), Barcelona, Spain, 2-5 July 2007. 
ICAO, 2006. Airborne collision avoidance system (ACAS) manual. Document 9863, International Civil Aviation Organization, Canada.

ICAO, 2014. Safety report. International Civil Aviation Organization, 2014 Edition, Canada.

Isaacson, D.R., Sadovsky, A.V., Davis, D., 2014. Tactical scheduling for precision air traffic operations: past research and current problems. Journal of Aerospace Information Systems 11(4), 234257.

Jensen, K. 1993. An introduction to the theoretical aspects of coloured petri nets. Springer Berlin Heidelberg, pp. 230-272.

Johnson, C., 2004. Final Report: review of the BFU Überlingen Accident Report. Contract $\mathrm{C} / 1.369 / \mathrm{HQ} / \mathrm{SS} / 04$, Eurocontrol.

Kochenderfer, M.J, Edwards M.W.M., Espindle, L.P., Kuchar, J.K., Griffith, J.D., 2010. Airspace encounter models for estimating collision risk. Journal of Guidance, Control, and Dynamics 33(2), 487-499.

Kristensen, L.M., Jensen, K., 2004. Specification and validation of an edge router discovery protocol for mobile ad hoc networks. Integration of Software Specification Techniques for Applications in Engineering, Springer Berlin Heidelberg, pp. 248-269.

Kuchar, J.K., Andrews, J., Drumm, A., Hall, T., Heinz, V., Thompson, S., Welch, J., 2004. A Safety Analysis Process for the Traffic Alert and Collision Avoidance System (TCAS) and See-andAvoid Systems on Remotely Piloted Vehicle. AIAA 3rd "Unmanned Unlimited" Technical Conference, Workshop and Exhibit, USA.

Kuchar, J.K., Yang, L.C., 2000. A review of conflict detection and resolution modelling methods. IEEE Transactions on Intelligent Transportation Systems 1(4), 179-189.

Ladkin, P.B., 2004. Causal analysis of the ACAS/TCAS Sociotechnical System. In: Proceedings of the 9th Australian workshop on Safety critical systems and software-Volume 47, Brisbane, Australia, pp.3-12.

Lee, R., Wolpert, D., 2012. Game theoretic modeling of pilot behavior during mid-air encounters. Decision Making with Imperfect Decision Maker, Springer Berlin Heidelberg, pp. 75-111.

McCallie, D., Butts, J., Mills, R., 2011. Security analysis of the ADS-B implementation in the next generation air transportation system. International Journal of Critical Infrastructure Protection $4(2), 78-87$.

Netjasov, F., Vidosavljevic, A., Tosic, V., Everdij, M.H.C., Blom, H.A.P., 2010. Stochastically and dynamically coloured Petri net model of ACAS operations. In: Proceedings 4th international conference on research in air transportation (ICRAT). Budapest, Hungary, 1-4 June 2010, pp. 449-456.

Netjasov, F., Vidosavljevic, A., Tosic, V., Everdij, M.H., Blom, H.A.P., 2013. Development, validation and application of stochastically and dynamically coloured Petri net model of ACAS operations for safety assessment purposes. Transportation Research Part C: Emerging Technologies 33, 167-195.

Nosedal, J., Piera, M.A., Ruiz, S., Nosedal, A., 2014. An efficient algorithm for smoothing airspace congestion by fine-tuning take-off times. Transportation Research Part C: Emerging Technologies 44, 171-184.

NTSB database, 2015. <http://www.ntsb.gov/_layouts/ntsb.aviation/month.aspx>, National Transportation Board, accessed on 7 March 2015.

Piera, M.A, Music, G., 2011. Coloured Petri net scheduling models: Timed state space exploration shortages. Mathematics and Computers in Simulation 82(3): 428-441.

Piera, M.A., Ramos, J.J., Moreno, R., Narciso, M., 2014. Causal simulation models for facing third millennium air transport sustainability. Simulation 90, 162-170.

Ruiz, S., 2013. Strategic trajectory de-confliction to enable seamless aircraft conflict management. Ph.D. Dissertation, Telecommunication and System Engineering Dept., Autonomous of Barcelona Univ., Barcelona, ES, 2013.

Salimifard, K., Wright, M., 2001. Petri net-based modelling of workflow systems: An overview. European journal of operational research 134(3), 664-676.

Tang, J., Piera, M.A., Ruiz, S., 2014. A causal model to explore the ACAS induced collisions. Proceedings of the Institution of Mechanical Engineers, Part G: Journal of Aerospace Engineering 228(10), 1735-1748. 
Wildmann, N., Ravi, S., Bange, J., 2014. Towards higher accuracy and better frequency response with standard multi-hole probes in turbulence measurement with remotely piloted aircraft (RPA). Atmospheric Measurement Techniques 7(4), 1027-1041.

Yousefi, A., Zadeh, A.N., 2013. Dynamic allocation and benefit assessment of NextGen flow corridors. Transportation Research Part C: Emerging Technologies 33, 297-310.

Zeitlin, A., Lacher, A., Kuchar, J.K., Drumm, A., 2006. Collision avoidance for unmanned aircraft: Proving the safety case (No. MP-060219). The MITRE Corporation and MIT Lincoln Laboratory, USA.

Zuniga, C.A., Piera, M.A., Ruiz, S., Del Pozo, I., 2013. A CD\&CR causal model based on path shortening/path stretching techniques. Transportation Research Part C: Emerging Technologies 33, 238-256. 